\title{
La construcción de secuencias cronológicas. Una aplicación estadística multivariante a la Edad del Hierro en el valle medio del Tajo
}

\section{The construction of chronological sequences. A multivariate statistical ap- proach to the Iron Age of the Middle Tagus valley}

\author{
Jorge DE TORRES RodríGUEZ \\ Universidad Complutense de Madrid \\ jorgedetorres@yahoo.es
}

Recibido: 15-04-2013

Aceptado: 08-05-2013

\begin{abstract}
RESUMEN
El artículo presenta un ejemplo del potencial de una herramienta de análisis estadístico como es el Análisis de Correspondencias para el procesado, presentación e interpretación de conjuntos de datos cualitativas poco sistematizados. Este método estadístico se aplica a la Edad del Hierro del valle medio del Tajo, una región en la que el aumento de la información arqueológica de los últimos 15 años no ha devenido en el establecimiento de secuencias cronológicas bien definidas para el periodo. A partir de los resultados del análisis multivariante y apoyado en las dataciones absolutas existentes y el registro arqueológico, se propone por primera vez una cronología general para el primer milenio a.C. en la región.
\end{abstract}

Palabras Clave: Arqueología protohistórica. Análisis estadístico. Valle del Tajo. Edad del Hierro. Carpetania.

\begin{abstract}
This paper shows the potential of a type of multivariate analysis (Correspondence Analysis) as a statistical tool useful to process, present and interpret qualitative information, especially when dealing with poorly systematized datasets. This statistical method is applied to the middle Tagus valley Iron Age, a region where the last decades' archaeological information improvement has not yet brought about a clear, well defined chronological sequence for the period. Multivariate Analysis results, combined with existing absolute datings and the archaeological record are used to propose the first general chronology for the $1^{\text {st }}$ millennium B.C. in this region.
\end{abstract}

KeY words: Protohistoric Archaeology. Statistical analysis. Tagus valley, Iron Age. Carpetania. 


\section{Introducción}

Hasta finales de la década de 1990, la protohistoria de la mitad oriental del valle medio del Tajo era una de las peor conocidas de la Península ibérica, una especie de vacío mal definido territorial, material y culturalmente con pocos yacimientos excavados y menos aún sistemáticamente publicados. Tan sólo se habían publicado las memorias de Las Madrigueras (Almagro 1969), Cerro Redondo (Blasco y Alonso 1985), Cerro de San Antonio (Blasco et al. 1991) o El Cerrón (Valiente 1994), y para el resto de yacimientos excavados la información oscilaba entre la relativamente abundante -caso, por ejemplo, de Palomar de Pintado (Ruiz et al. 2004; Pereira et al. 2003; Pereira et al. 2001)- y breves comunicaciones o artículos. Esta situación ha cambiado significativamente en los últimos quince años, sobre todo gracias a la arqueología de gestión que ha multiplicado de manera exponencial el número de yacimientos conocidos y publicados total o parcialmente. La Edad del Hierro del valle medio del Tajo - una zona que corresponde aproximadamente a la Comunidad de Madrid y la mitad oriental de Toledo - ha vivido una verdadera explosión de información que se ha plasmado en todos los ámbitos: prospecciones, proyectos de investigación en yacimientos como Llano de la Horca o Dehesa de la Oliva, exposiciones (VV.AA. 2002; Quero et al. 2005; Ruiz et al. 2012), jornadas y seminarios dedicados íntegra o parcialmente a este periodo (VV.AA. 2007; VV.AA. 2008; VV.AA. 2010), musealización de yacimientos como Miralrío (Laguna del Capillo) en Rivas - Vaciamadrid, publicación de libros de síntesis (Carrasco 2007; Pereira 2007) y de monográficos (VV.AA. 2007), etc.

Por desgracia, este aumento cuantitativo de la información no se ha reflejado en el establecimiento de secuencias arqueológicas bien definidas, con cronologías ajustadas e interpretaciones elaboradas de los procesos económicos, políticos y sociales desarrollados a lo largo del primer milenio a.C. en la región. Salvo algunas excepciones (Urbina 1997, 1998; 2000), la construcción del conocimiento arqueológico en el valle medio del Tajo se ha producido más como acumulación de datos de calidad muy desigual que a través del desarrollo de metodologías e hipótesis de trabajo que permitan ordenar e interpretar el registro. Por otra parte, el marco cronológico utilizado es excesivamente simple y con unos hitos que se parecen sospechosamente a los de regiones cercanas, como si se hubieran extrapolado desde zonas más conocidas. Así, se acepta una fecha en torno al 800 a.C. para el comienzo de la Edad del Hierro en el valle (Blasco 2007, 69-70) y el siglo V a.C. como el momento de transición a la Segunda

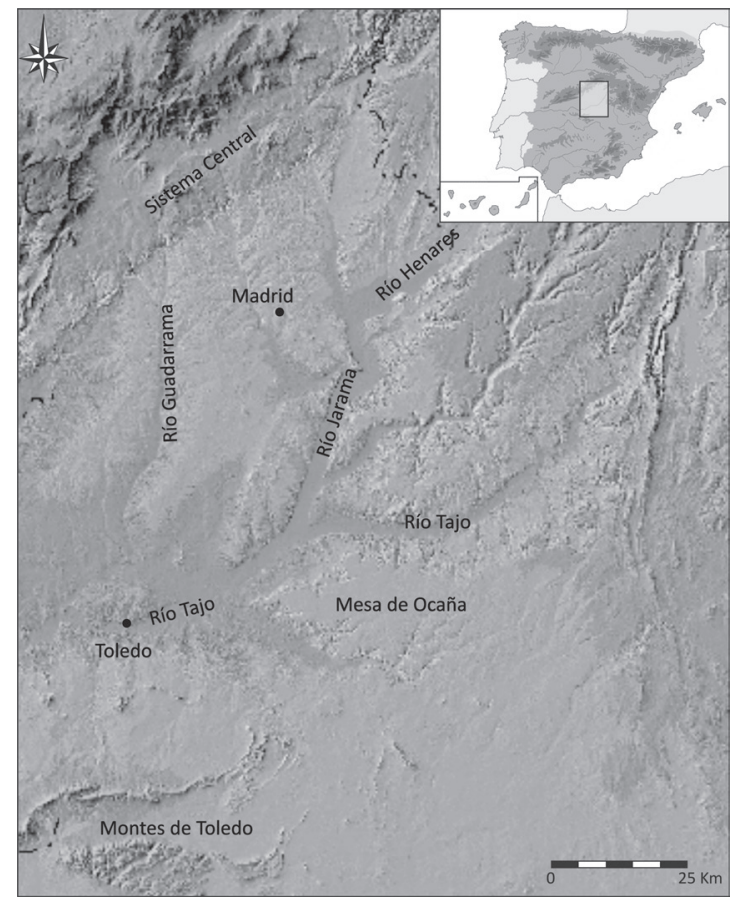

Fig. 1.- Zona de estudio. El valle medio del Tajo.

Edad del Hierro (Ruiz 2007: 43). Este marco general se acepta de manera acrítica, sin profundizar mucho más en las características de cada periodo, el ritmo y velocidad de las transformaciones materiales perceptibles en el registro arqueológico y sus posibles implicaciones socioeconómicas. El resultado es que, a día de hoy, no existe una secuencia cronológica clara para la Edad del Hierro de la zona oriental del valle medio del Tajo y que las adscripciones cronológicas de los yacimientos se realizan con una gran indefinición, apoyadas en fósiles directores -cerámica a torno, piezas de importación, etc.- que ni han sido objeto de estudio sistemático ni sirven para proponer una interpretación histórica del periodo.

El principal objetivo de este artículo es tratar de superar esta situación para proponer una secuencia cronológica general para la Edad del Hierro en la región, ordenando, en la medida de lo posible, los yacimientos conocidos y los principales elementos de cultura material recogidos en ellos. El procesado del registro necesario para realizar esta secuencia presenta, sin embargo, algunos problemas. La ausencia de publicaciones íntegras de la mayoría de las excavaciones provoca que a menudo se disponga tan sólo de información cualitativa -esto es, la presencia o no de determinados objetos-, que esta información presente una calidad muy variable y 
que en general se encuentre poco sistematizada. En estas condiciones es imposible desarrollar una metodología al uso basada en el estudio de estratigrafías bien estudiadas con cronologías ajustadas, y se hace necesario optar por caminos alternativos que permitan desbloquear una situación que, hoy por hoy, no tiene visos de mejorar.

Para el análisis de correspondencias se ha utilizado la práctica de totalidad de yacimientos excavados para los que se dispone de un mínimo de información, y en el caso de que existieran varias fases, se han seguido los criterios y las descripciones realizadas por los autores de las excavaciones. En cuanto al proceso de selección de las variables ha sido bastante complejo, aunque en líneas generales, podrían definirse tres grupos: las asociadas a las características topográficas y materiales de los asentamientos, elegidas por observación de los yacimientos de la región; las relacionadas con la cerámica de tipo ibérico - decoraciones, tipos, etc. - y fíbulas, para cuya selección nos apoyamos en los trabajos de Ruiz y Molinos (1993), Bonet y Mata (2008), Urbina (1997) y González (1999); y las que corresponden a piezas de importación como la cerámica ática, la de barniz rojo o las cuentas de pasta vítrea. Como es lógico, la selección de variables y yacimientos tiene un fuerte carácter subjetivo y aunque hemos tratado de que sean variables eficaces como herramientas de análisis estadístico todas ellas están sujetas a discusión. De hecho y como veremos en las conclusiones, algunas de ellas se demostraron poco operativas.

La herramienta elegida para realizar esta aproximación es el denominado Análisis de Correspondencias (AC), una técnica estadística plenamente consolidada como herramienta de análisis e interpretación en nuestra disciplina (Ringrose 1992, Jensen y Nielsen 1997a), para su introducción en la arqueología española, ver Fernández y García 1991, Guinea y Heras 1991), utilizada generalmente para detectar seriaciones cronológicas y agrupaciones significativas de datos. Sintetizando mucho, este análisis multivariante permite analizar las relaciones existentes entre variables de tipo cualitativo y los casos asociados a estas variables -sean tumbas, estratos, yacimientos, etc., en función de su mayor o menor ocurrencia mutua. Ordena estos datos en unos ejes de dos o tres coordenadas mostrando las distancias relativas entre las diferentes unidades y variables, correspondiendo una mayor distancia a una mayor diferencia entre casos/variables y viceversa. Esta mayor cercanía o lejanía entre casos variables puede tener diversas connotaciones - cronológicas, sociales, materiales, etc. - y corresponde al arqueólogo interpretar la distribución obtenida a través del análisis. Cuando el $\mathrm{AC}$ representa una seriación, ésta suele verse reflejada en el gráfico adoptando una forma de parábola, y la regularidad de la misma reflejará la mayor o menor perfección de la seriación, representando cada uno de los dos extremos de la parábola los casos más diferentes de toda la distribución. Esa diferencia no es explicada per se y debe ser interpretada por el arqueólogo. Puede tratarse de una oposición entre más antiguo y más moderno, pero la seriación también podría ser social, marcando las diferencias entre pobre/ rico, masculino/ femenino, etc.

El AC es actualmente una de las herramientas más potentes de análisis cualitativo en arqueología, gracias a las posibilidades de interpretación que se derivan de poder relacionar de manera conjunta un número muy elevado de variables y casos, imposible de aprehender a través de la simple observación visual o univariante. En nuestro caso, hemos utilizado 75 yacimientos (o fases de yacimientos) y 99 variables que hemos considerado relevantes para establecer una secuencia temporal que relacione cronológicamente yacimientos y variables. En un resultado ideal los yacimientos estudiados (o más bien, las diferentes fases de los yacimientos) se agruparían de manera coherente de acuerdo a variables similares (materiales asociados a una etapa cultural concreta, por ejemplo) y se distribuirían desde los más antiguos a los más modernos, esto es, desde la transición entre el Bronce Final y el Hierro I hasta el cambio de era. El sistema es muy parecido a la seriación realizada mediante el análisis multivariante llamado Escalograma multidimensional (Multidimensional Scaling) (Kendall 1971), aunque, a diferencia de éste, ordena tanto las unidades (contextos arqueológicos) como las variables que los definen.

Como hemos dicho antes, el AC no pone un valor a la distancia o cercanía existente entre dos casos o variables, de manera que la distribución obtenida debe ser contrastada con las dataciones absolutas $\left(\mathrm{C}_{14}, \mathrm{TL}\right.$, etc. $)$ existentes y con las cronologías aceptadas para las variables, que de nuevo en un modelo perfecto deberían imbricarse de manera coherente en el orden en que se han colocado los yacimientos representados. Con objeto de homogeneizar el conjunto de dataciones absolutas existente, que presenta una enorme variabilidad - incluyendo algunas muestras sin calibrar -, se ha optado por recalibrar todas usando el programa CalPal y presentar los intervalos en los diferentes gráficos con objeto de visualizar mejor las cronologías propuestas por cada muestra. En el caso de las dataciones de Termoluminiscencia, se ha representado el intervalo de probabilidad para que puedan ser comparadas con las dataciones radiocarbónicas. En el Anexo III de mi Tesis Doctoral (Torres 2012) puede consultarse una 


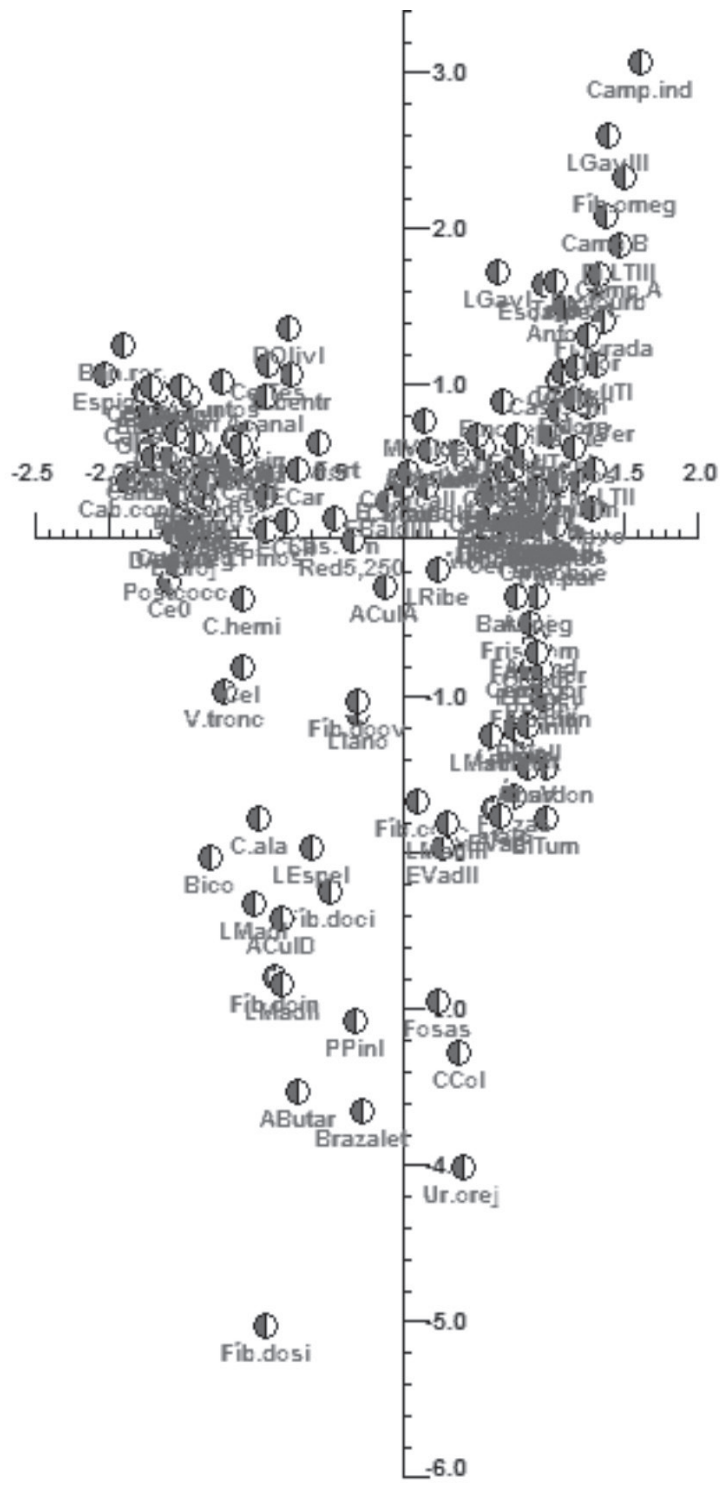

Fig.a 2.- Resultados básicos del análisis multivariante. Véase una expansión de las diferentes partes del gráfico en las restantes figuras del artículo.

discusión completa sobre el corpus de dataciones absolutas del valle medio del Tajo. Para realizar el análisis el programa utilizado ha sido el WinBASP (Bonn Archaeological Statistical Package) versión 5.4.3 desarrollado por la Universidad de Colonia. Se trata de un software gratuito que puede ser descargado en la página www.uni-koeln.de y que permite realizar, además de Análisis de Correspondencias, seriaciones, análisis de componentes principales y matrices estratigráficas.

\section{Resultados generales}

El gráfico inicial del $\mathrm{AC}$ (fig. 2) presentaba una forma con tendencia parabólica, con dos grandes concentraciones de casos y variables en los dos extremos y una zona menos clara en el centro. Como hemos comentado arriba, esta distribución parabólica -aun irregular- es un indicio de la existencia de una seriación cronológica en el gráfico, mucho más clara en su mitad derecha. El análisis de yacimientos y variables permite identificar seis conjuntos de yacimientos ordenados de mayor a menor antigüedad (fig. 3) en función de los materiales arqueológicos localizados. En el extremo izquierdo se sitúan los yacimientos y variables asociadas a la Primera Edad del Hierro, mientras que a la derecha se identifican los yacimientos de la Segunda Edad del Hierro, con una zona intermedia interpretada como "de transición" en el centro de los ejes. El sentido de la parábola es por tanto cronológico, con los casos y variables más antiguos y más modernos en los dos extremos de sus ramas.

Además de esta connotación cronológica, el análisis de correspondencia detectó otro nivel de interpretación en la muestra, relacionado con el tipo de yacimiento analizado. Así, en la mitad superior del gráfico se disponen los asentamientos (independientemente de su adscripción cronológica), mientras que en la mitad inferior se han representado las necrópolis. Por lo tanto, el eje horizontal es cronológico y el eje vertical es podría interpretarse de manera «sociológica», separando los yacimientos según su funcionalidad. Esta dicotomía es la que hace muy imprecisa la zona central de la parábola y es la principal anomalía dentro del análisis, pero tiene una explicación relativamente sencilla y muy coherente con la realidad del registro arqueológico.

Esta explicación se apoya en la constatación de que en los enterramientos se depositan generalmente objetos especiales, bien por su excepcionalidad, bien por su número. Ejemplos muy evidentes en nuestro caso son las fíbulas de doble resorte y anulares o las cuentas de pasta vítrea, aparecidas mayoritariamente en contextos funerarios. Estas diferencias influyen en la disposición de las necrópolis en el gráfico, que aparecen separadas y asociadas a una cultura material específica. La asociación de determinados materiales a las necrópolis se aprecia claramente en las necrópolis de la Segunda Edad del Hierro (que forman un conjunto muy compacto) y peor en las necrópolis de la Primera Edad del Hierro (más dispersas). Por otra parte, la creación de estos dos grupos (necrópolis y asentamientos) distorsiona la localización de algunas variables que aparecen en ambos tipos de yacimientos y que quedan así en una especie de "tierra de nadie". Sin embargo, esta 


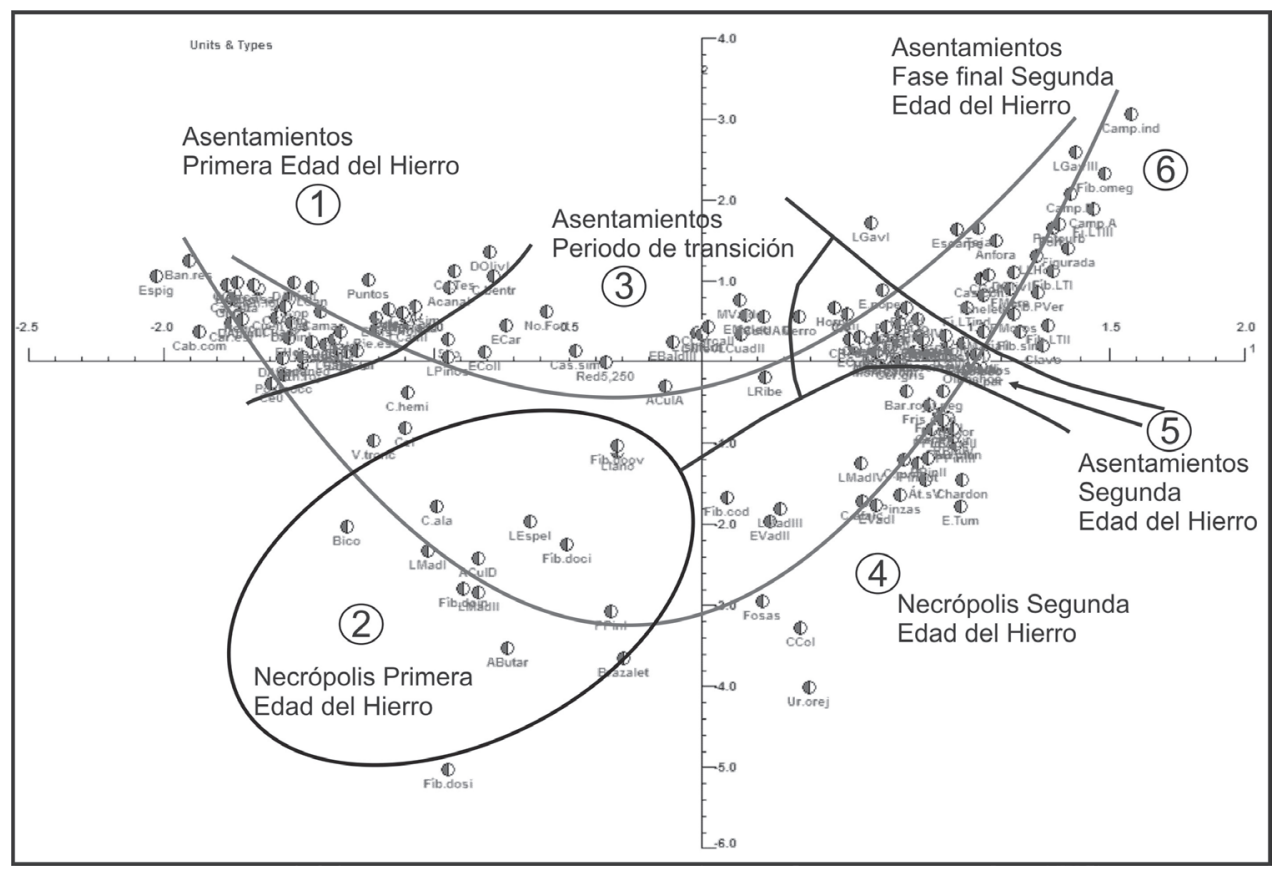

Fig. 3.- Interpretación cronológica del análisis multivariante, con las seis zonas cronológicas identificadas. Ver explicaciones de los códigos en los apéndices al final del artículo.

individualización de las necrópolis como grupo no impide su correcta asignación cronológica dentro del gráfico ya que se encuentran coherentemente dispuestas dentro de la tendencia general de la secuencia. Así, las necrópolis de la Primera Edad del Hierro aparecen en la mitad izquierda del gráfico, cerca de los asentamientos de este periodo, y lo mismo ocurre con las necrópolis de la Segunda Edad del Hierro. La separación de las necrópolis del conjunto de yacimientos puede tener también unas connotaciones materiales interesantes, ya que podría ayudar a identificar una cultura material específica de contextos funerarios en el valle medio del Tajo. La individualización de las necrópolis no supone un problema interpretativo sobre el gráfico, pero sí introduce un elemento distorsionante en el mismo que debe ser tenido en cuenta a la hora de valorar la secuencia.

\section{Discusión de la secuencia}

\subsection{La Primera Edad del Hierro}

Como corresponde al momento más antiguo de la secuencia, se localiza en uno de los extremos de la parábola, en el cuadrante superior izquierdo del gráfico (fig. 4). Constituye un grupo compacto de yacimientos bien ordenados, en el que las variables características de la transición entre el Bronce Final y la Primera Edad del Hierro (decoraciones cerámicas como la excisión o el boquique) se sitúan en la parte superior izquierda del conjunto, -el comienzo de la secuencia- mientras que aquellas más modernas -como la que marca la aparición de cerámica a torno- se sitúan a la derecha. En la zona central, correspondiente al periodo pleno de la Primera Edad del Hierro, los casos y las variables parecen agruparse en dos conjuntos asociados a dos de los yacimientos más conocidos de este periodo: Cerro de San Antonio y La Capellana.

La distribución de casos y variables muestra una tendencia general coherente y confirma en gran medida los datos propuestos desde las aproximaciones arqueológicas apoyadas exclusivamente en el estudio de los materiales. En cuanto a la cronología del periodo (fig. 5), sólo disponemos de dataciones absolutas para cuatro yacimientos (Las Camas, Ecce Homo I, Cerro de San Antonio y La Capellana). En el caso de las dataciones de $\mathrm{C}_{14}$, algunas presentan una clara incoherencia con la posición de los yacimientos en el gráfico (Las Camas y Ecce Homo), ya que se sitúan en un intervalo entre el 1500 1000 a.C., con una clara falta de concordancia con la cultura material recuperada en aquéllos. En Las Camas, esta discordancia es aceptada por sus ex- 


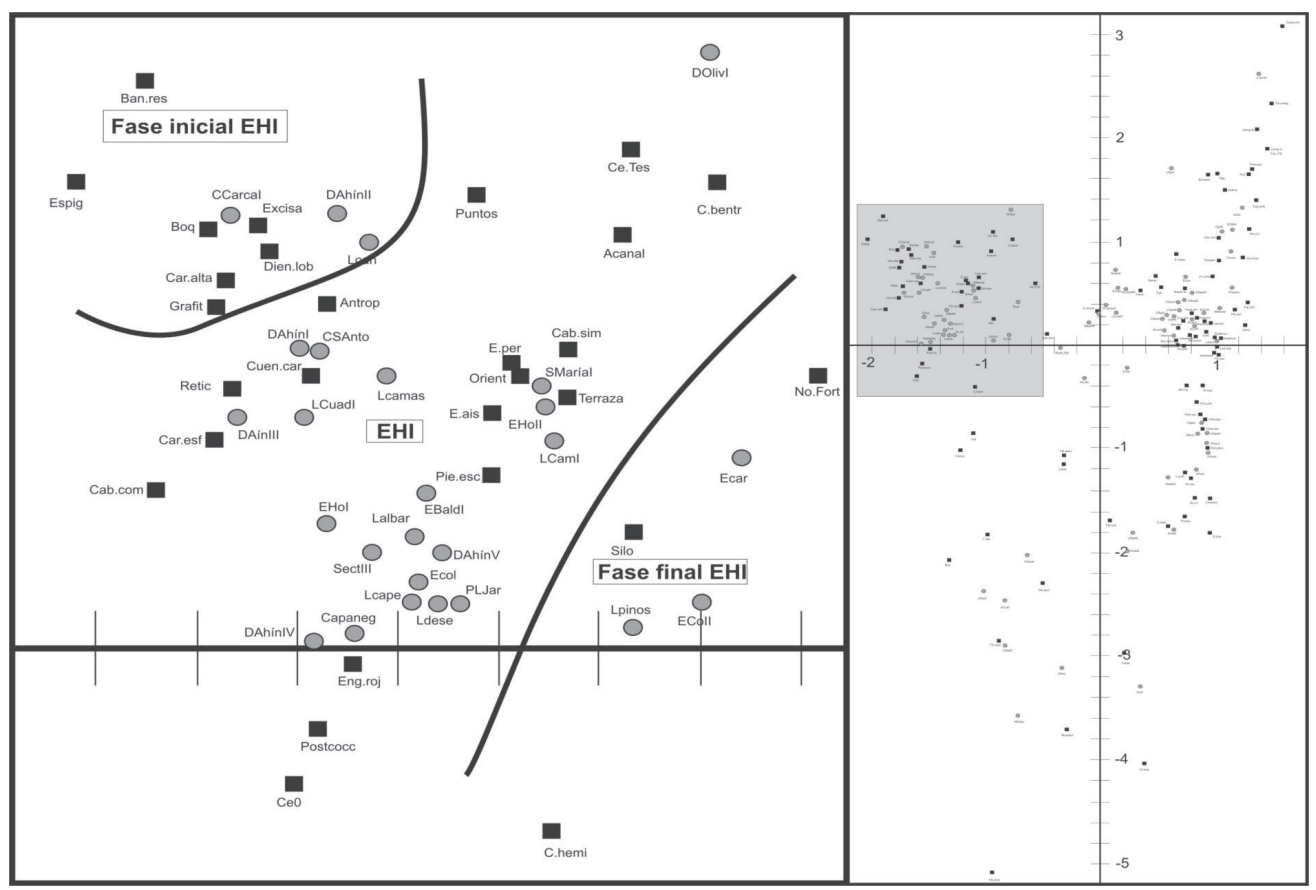

Fig. 4.- Distribución de yacimientos (círculos grises) y variables (cuadrados negros) en la zona identificada como perteneciente a la Primera Edad del Hierro (izquierda). Localización de la zona de la Primera Edad del Hierro en el gráfico general (derecha).

cavadores, que tratan de conciliar ambas posturas asumiendo que los materiales recuperados corresponden al momento final de la ocupación que fecharían como muy tarde en el siglo VIII a.C. (Urbina et al. 2007: 70). Es cierto que el yacimiento de Las Camas presenta materiales considerados como arcaicos - cerámica excisa, vasijas con carena alta, bandas reservadas, etc.- pero también elementos considerados como pertenecientes al periodo pleno de la Primera Edad del Hierro (cerámica postcocción, engobe rojo, cuencos carenados con decoración incisa). Con los datos disponibles es imposible dilucidar la dicotomía entre los resultados del $\mathrm{C}_{14} \mathrm{y}$ las evidencias del registro material. En cuanto a la otra datación disponible, la realizada para Ecce Homo, la muestra debe descartarse al proceder de dos basureros diferentes (Almagro y Fernández-Galiano 1980: 125). En este caso además los materiales también presentan problemas, ya que corresponden a las estructuras de las primeras campañas de excavación, en las que se recuperaron muchos materiales revueltos en basureros. De este modo, las dataciones radiocarbónicas no aportan ningún elemento de juicio para afinar la cronología relativa ofrecida por el gráfico, pero dados los problemas de ambas dataciones, sin embargo, tampoco no consideramos que puedan desautorizar la tendencia general marcada por el AC.
En cuanto a los resultados de termoluminiscencia y su relación con las dataciones radiocarbónicas existentes, ofrecen uno de los principales problemas de interpretación de toda la serie. En el caso del Cerro de San Antonio (Rubio y Blasco 2000), el intervalo de coincidencia entre las tres dataciones ofrece un resultado en torno al siglo VII a.C., mientras que para La Capellana (Blasco et al. 1993) el intervalo central de las tres dataciones consideradas aceptables se sitúa a finales del siglo VI a.C., fecha que parece coherente con los materiales arqueológicos y con la posición del asentamiento en el gráfico. El panorama cronológico abarcaría, grosso modo, la segunda mitad del siglo VII a.C. y el siglo VI a.C. El problema de estas dataciones es su absoluta falta de coherencia con las dataciones radiocarbónicas por otra parte, muy antiguas. Con los datos disponibles tan sólo puede avanzarse que Cerro de San Antonio parece ser más antiguo que la Capellana -algo que también refleja el análisis multivariantey que podría enlazar con otros asentamientos aún más antiguos que se situarían en el comienzo de la Edad del Hierro.

Pese a los problemas planteados por las dataciones absolutas y a algunas anomalías en la distribución -asociadas a la escasez de información respecto de algunos yacimientos o al efecto causado por la 


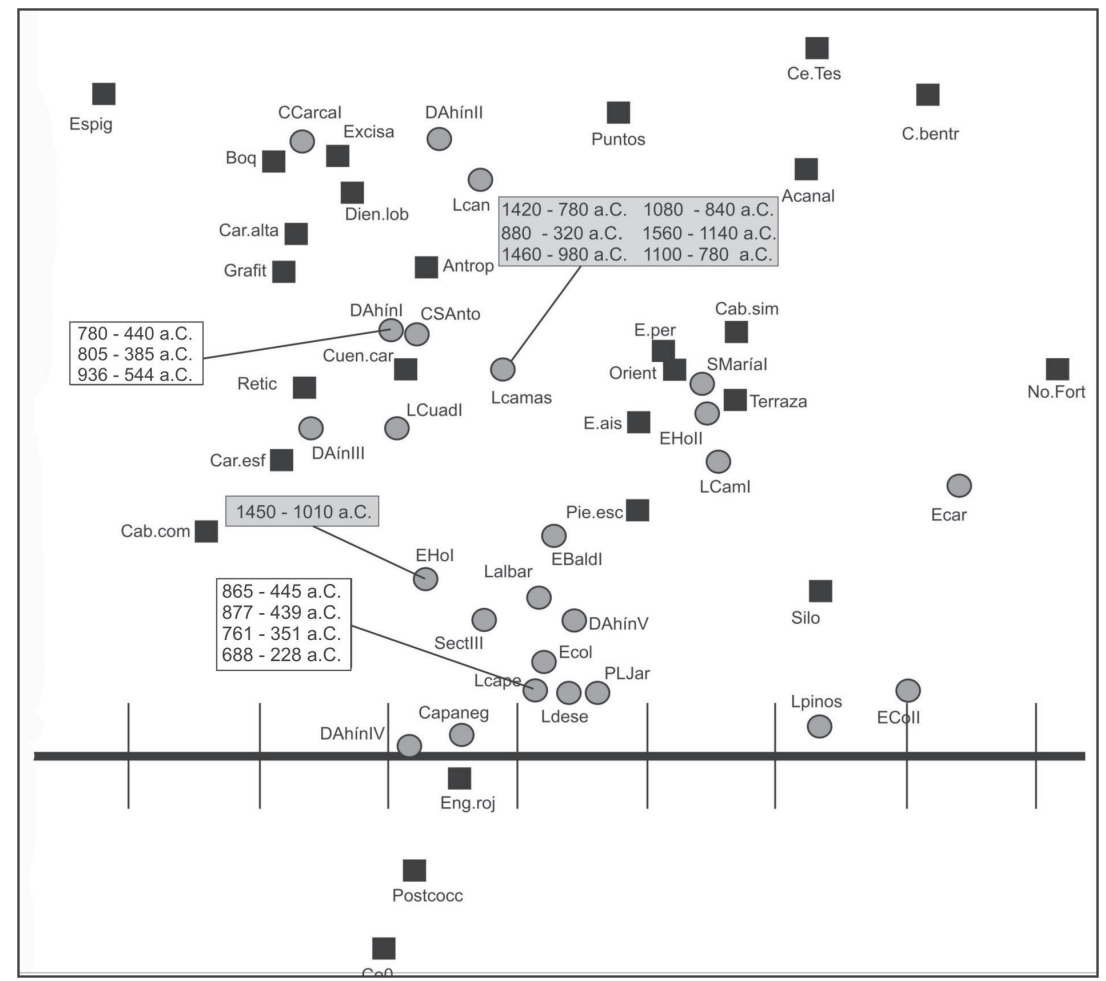

Fig. 5.- Dataciones absolutas los yacimientos de la Primera Edad del Hierro representados en el análisis. En gris, dataciones radiocarbónicas (calibradas a $2 \sigma$ ). En blanco, dataciones por termoluminiscencia.

separación que hace el análisis entre asentamientos y necrópolis- los datos existentes nos permiten establecer una propuesta de secuencia para el periodo, quese iniciaría con yacimientos con fuertes reminiscencias de la cultura material del Bronce Final, visibles especialmente en las tradiciones decorativas y en algunas formas cerámicas como los cuencos de carena alta. Los dos yacimientos que parecen representar mejor este momento son Dehesa de Ahín II y Camino de las Cárcavas y, en menor medida, La Cantueña. Cronológicamente el periodo es difícil de situar, ya que no contamos con dataciones absolutas por lo que las referencias deben buscarse con respecto al periodo central de la Primera Edad del Hierro que podría datarse, de manera un poco imprecisa sobre todo en su comienzo, en torno al siglo VIII a.C.

La fase plena de la Primera Edad del Hierro parece mostrar dos momentos bastante claros, el primero de ellos asociado a yacimientos como Cerro de San Antonio, la Dehesa de Ahín I y Las Camas. La distancia entre los yacimientos apunta a una evolución rápida de la cultura material (Jensen y Nielsen 1997a: 48). El principal problema de definición de este momento más antiguo es cronológico. Por una parte, se aprecian fuertes similitudes en la cerámica de los yacimientos pertenecientes a este horizonte (Urbina et al. 2007; Rojas et al. 2007: 61, 92). Sin embargo, mientras que yacimientos como Dehesa de Ahín o Cerro de San Antonio son encuadrados en torno al siglo VII a.C. por los responsables de su excavación (Rojas et al. 2007: 103, Rubio y Blasco 2000: 229), las fechas radiocarbónicas de Las Camas son mucho más antiguas, centradas en torno al cambio de milenio a.C., y coherentes con algunos rasgos de la cultura material como las plantas de las grandes cabañas documentadas en el yacimiento o las evidencias metalúrgicas con reminiscencias del Bronce Final (Urbina et al. 2007: 73).

La argumentación de los autores de la excavación para justificar las fechas radiocarbónicas presenta algunos problemas, entre ellos, el ya citado uso de fechas procedentes del yacimiento de Ecce Homo, cuando menos dudosas. Finalmente, se propone una fecha en torno al siglo IX a.C. y la primera mitad del S. VIII a.C. para el asentamiento, al asumir que las muestras son de vida larga y que por tanto podrían encuadrarse en el extremo más moderno del intervalo considerado (Urbina et al. 2007: 67). Aunque la cronología de los comienzos 


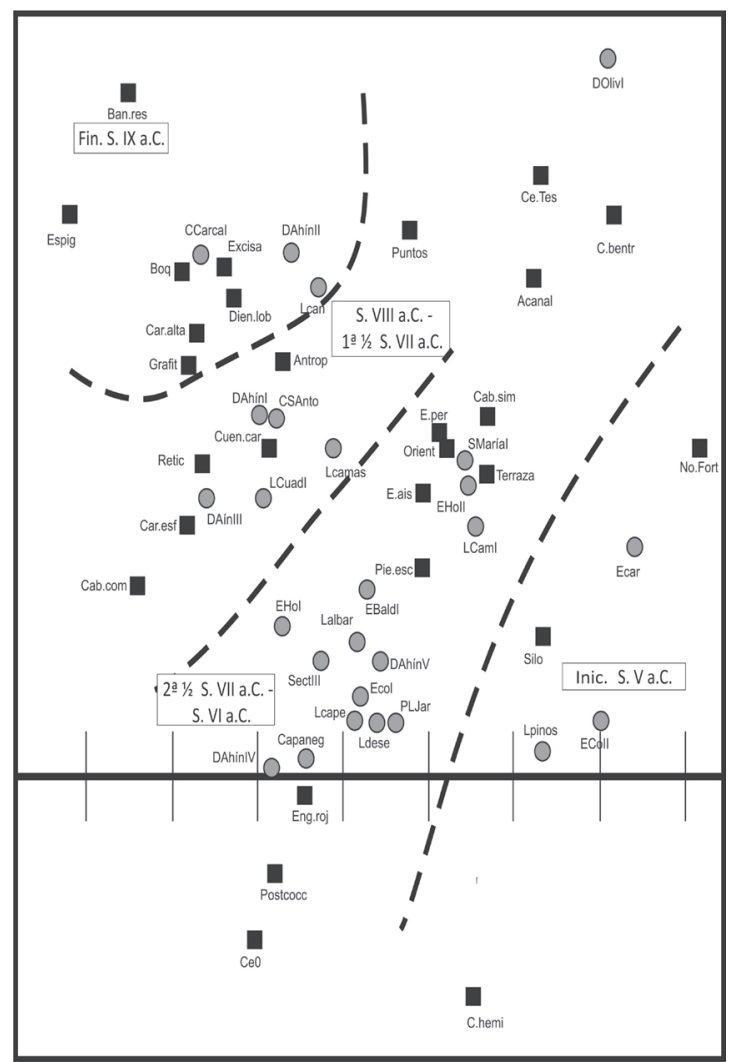

Fig. 6.- interpretación cronológica de la Primera Edad del Hierro. Los cuadrados negros corresponden a variables, los círculos grises, a yacimientos.

de la Edad del Hierro en el valle medio del Tajo sigue estando poco clara, las diferencias cronológicas entre asentamientos con una cultura material muy similar, como Las Camas o Cerro de San Antonio podrían obedecer simplemente a la mayor antigüedad de un yacimiento respecto de otro, en un mismo momento cultural. Así, Las Camas sería un yacimiento antiguo -en torno al siglo VIII a.C.- de este primer momento de la Primera Edad del Hierro, mientras que Cerro de San Antonio y Dehesa de Ahín corresponderían a un momento más avanzado, en torno al siglo VII a.C., momento en el que se da la mayor coincidencia de los intervalos de Termoluminiscencia.

El segundo momento dentro de la Primera Edad del Hierro, representado por yacimientos como La Capellana, Dehesa de Ahín IV y V, El Colegio o Puente largo del Jarama, está mucho mejor definido cronológica y materialmente. En esta fase los yacimientos aparecen más agrupados en el análisis, algo que podría interpretarse como un síntoma de mayor estabilidad (Jensen y Nielsen 1997a: 48), y se hace patente el comienzo de las influencias procedentes del mundo del suroeste y mediterráneo en la aparición de variables como los objetos orientalizantes y la cerámica de engobe rojo asociada a este conjunto de asentamientos. Asimismo, este periodo puede relacionarse con la aparición de las necrópolis de incineración, cuya cronología hasta el momento no parece superar el siglo VI a.C. Así pues, el horizonte cronológico de este segundo periodo podría datarse entre mediados/ finales del siglo VII a.C. y el siglo VI a.C.

El final de la Primera Edad del Hierro vendría caracterizado por tres yacimientos: Los Pinos, El Caracol y El Colegio II. El primero de ellos presenta cerámicas a torno dentro de un contexto de cabañas, mientras que los otros presentan construcciones en materiales no perecederos, si bien de escasa entidad. Parece sin embargo que el elemento que marca el comienzo del cambio (en cuanto a cultura material se refiere, por lo menos, y respecto a los datos que muestra el AC) es la introducción del torno y no la construcción de estructuras construidas en materiales no perecederos, ya que tanto en La Capellana como Puente Largo del Jarama se han documentado este tipo de estructuras y sin embargo aparecen claramente agrupados como yacimientos de la Primera Edad del Hierro plena. En cuanto a la cronología, por su posición en el gráfico y su cercanía al grupo de La Capellana creemos válida una fecha en torno al final del siglo VI a.C. o comienzos del siglo V a.C., ajustando un poco las propuestas tradicionales que sitúan de manera genérica la entrada de la cerámica a torno en la región en el siglo V a.C. (Valiente 1993; Urbina 1997: 533).

La progresión cronológica ha sido resumida en la figura 6. Es necesario recordar, en primer lugar, que las separaciones no son estancas -algo que se ha querido visualizar a través de líneas discontinuasy que el AC no representa distancias cronológicas, sino sólo similitudes o diferencias culturales, por lo tanto las distancias cronológicas no tienen por qué coincidir con distancias equivalentes en el gráfico. Esto obedece a las propias características del tipo de análisis estadístico, a su forma de visualización a través de ecuaciones de tipo logarítmico, que distorsionan la representación y a las características de la muestra. Finalmente, parece obvio remarcar que las fechas propuestas no son rígidas ya que no existen evoluciones perfectas en la cultura material de ninguna sociedad. Estas fechas no pretenden ser más que un esquema general de la secuencia del registro arqueológico en la región, y como hemos visto presentan algunos problemas de datación en la fase más antigua de la Primera Edad del Hierro.

En el caso de las variables las precauciones son idénticas. Que una variable como el engobe rojo 
aparezca en un periodo concreto no implica que esa cerámica tenga, estrictamente, esa cronología. Simplemente indica que es en ese momento cuando es más característica y se encuentra asociada a un mayor número de asentamientos. No excluye que pueda aparecer - de manera menos destacada, eso sí- en yacimientos de etapas posteriores o anteriores. Como ocurre siempre en estadística (por correctos que sean los análisis), lo que se define son tendencias más o menos firmes, y con ese espíritu deben ser interpretados tanto los resultados del gráfico como sus asociaciones cronológicas

\subsection{Necrópolis de la Primera Edad del Hierro}

El grupo 2 del análisis, caracterizado por agrupar a todas las necrópolis de la Primera Edad del Hie- rro, se sitúa en el cuadrante inferior izquierdo del gráfico (fig. 7, izquierda) y tiene unas características muy diferentes a las de los asentamientos de ese mismo periodo. En primer lugar, es un conjunto mucho menor y presenta una gran dispersión, lo que dificulta la detección de los criterios de ordenación de los yacimientos y sus variables asociadas. En segundo lugar, y al igual que ocurría en el otro grupo, la presencia de objetos que también se localizan en asentamientos hace que algunas variables se encuentren desplazadas y sea difícil detectar las relaciones entre variables y yacimientos. La concentración de necrópolis (figura 7, derecha) ofrece pocos elementos de análisis. Tan sólo hay seis yacimientos que correspondan a esta categoría (Arroyo Culebro D, Las Madrigueras I y II, Arroyo Butarque, Palomar de Pintado I y Las Esperillas I), y

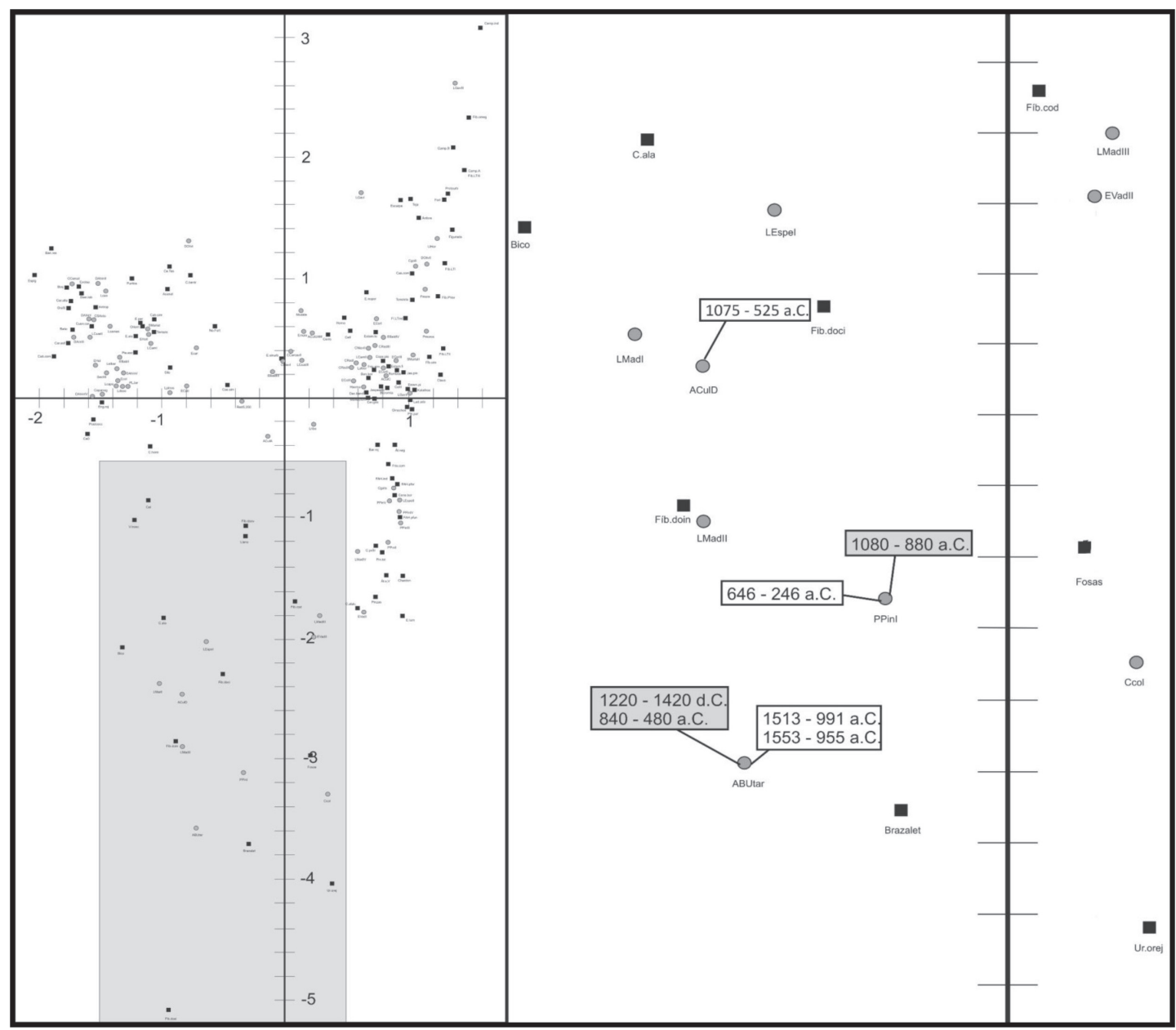

Fig. 7.- Localización de las necrópolis de la Primera Edad del Hierro en el gráfico general (izquierda) y ampliación de las necrópolis (círculos grises) y variables (cuadrados negros) de la zona estadísticamente más significativa (derecha). Las dataciones radiocarbónicas (calibradas a $2 \sigma$ ) aparecen recuadradas en gris, las de termoluminiscencia, en blanco. 
las variables a ellos asociadas parecen poco claras. De ellas las más evidentes son las fíbulas de doble resorte, de las que tres tipos están asociados a las necrópolis. Esta situación no hace más que evidenciar la presencia mayoritaria de este tipo de objetos en contextos funerarios. También los cuencos con borde en forma de ala, los brazaletes de bronce y los perfiles bicónicos se asocian a este tipo de yacimientos.

Dentro de los problemas que presenta una muestra tan pequeña, algunos indicadores apuntan a una correcta ordenación cronológica de los mismos. Así, la única necrópolis del periodo que presenta cerámica a torno (Arroyo Butarque) se encuentra al final de la serie, y las dos necrópolis más cercanas (Palomar de Pintado I y Las Madrigueras II) presentan algunos objetos que insinúan que podrían pertenecer también a un momento avanzado del periodo. Así, en Palomar de Pintado I se documentó un cuchillo afalcatado de hierro, mientras que en Las Madrigueras II se recogieron cuentas de pasta vítrea.

Por el contrario, Arroyo Culebro D y Las Madrigueras I parecen presentar características algo más arcaicas. Arroyo Culebro presenta características muy similares a Arroyo Butarque, incluida la presencia testimonial de hierro, pero carece de piezas a torno y sí de una que podría ser clasificada como engobe rojo. Siendo ligeramente más antigua que Arroyo Butarque, debe ser situada cronológicamente dentro del siglo VI a.C. Finalmente, el caso de Las Esperillas I, con valores muy dispares, resulta muy dudoso por la falta de claridad en la información disponible de este yacimiento, del que tan sólo se conoce la descripción de doce tumbas.

Las dataciones existentes no ayudan a afinar el panorama cronológico de la secuencia. La datación de TL de Arroyo Culebro D $(2750 \pm 275)$ (Penedo et al. 2002b: 309) parece muy elevada y tiene una desviación estándar demasiado amplia, y los materiales (cerámicos y metálicos) no coinciden con los documentados en asentamientos del siglo VIII a.C. Algo parecido ocurre con las dataciones de TL de Arroyo Butarque (Blasco et al. 2007), claramente erróneas ya que ofrecen fechas centrales en torno al siglo XIII a.C. incompatibles con la presencia de cerámica a torno y fíbulas de doble resorte. Finalmente, la datación de Palomar de Pintado (Pereira, com. pers) ofrece una fecha central en torno al siglo IV a.C. excesivamente moderna. Tampoco las dataciones radiocarbónicas aportan mucha información, ya que de las tres conocidas una (Arroyo Butarque) está contaminada ofreciendo una fecha de 1220 - 1420 d.C. calibrada a $2 \sigma$ (Blasco et al. 2007), mientras que otra (Palomar de Pintado I) presenta muchas dudas al ofrecer una cronología muy alta $(1080$ - 880 a.C. calibrada a $2 \sigma)$ para la introduc- ción de objetos de hierro en el valle medio del Tajo (Pereira et al. 2003: 162). La segunda datación de Arroyo Butarque (Blasco et al. 2007: 229) parece más ajustada ( 840 - 480 a.C. calibrada a $2 \sigma)$, pero su coincidencia con la meseta de Hallsttat impide afinar la cronología. Esta meseta, denominada así por el yacimiento centroeuropeo con cuya cronología coincide, es una zona muy plana en la curva de calibración del carbono 14, haciendo que las fechas en torno al 2450 BP ofrezcan de manera recurrente resultados entre 800 y 400 a.C., independientemente de la calidad de las medidas. La meseta de Halsttat hace que ajustar la datación radiocarbónica de los comienzos de la Edad del Hierro sea poco menos que imposible. En este caso, y según nuestra opinión, ninguna de las dataciones ofrece garantías suficientes como para mejorar la cronología de un periodo tan corto como el que parecen representar los materiales de las necrópolis.

Con los datos aquí presentados y los resultados alcanzados en el análisis parece clara la existencia de dos momentos arqueológicos muy cercanos en el tiempo pero bien definidos. El primero de ellos -situado en pleno siglo VI a.C.- está representado por Arroyo Culebro D y las Madrigueras I, con presencia de cuencos hemiesféricos, engobe rojo, vasos troncocónicos y fíbulas de doble resorte con puente de cinta, relacionados con asentamientos como la Capellana y que podrían datarse a comienzos del siglo VI a.C. El segundo momento, en torno al último cuarto del siglo VI y los comienzos del siglo V a.C., estaría caracterizado por Arroyo Butarque, Las Madrigueras II y Palomar de Pintado. Mantiene elementos anteriores pero introduce algunos objetos claramente adscritos a la fase final de la Primera Edad del Hierro, como son los cuencos de ala, las vasijas de perfil bicónico y sobre todo la presencia de los primeros objetos a torno en Arroyo Butarque o de cuentas de pasta vítrea amarilla (consideradas las más antiguas y datadas en torno al siglo VI a.C.) (Ruano 1996) en Las Madrigueras II. Aunque el hierro está presente en los dos momentos, en la primera su presencia es testimonial mientras que en la segunda aparece de manera más abundante.

La cronología de aparición de las necrópolis de incineración en el valle medio del Tajo vendría situada por tanto en el siglo VI a.C., de manera bastante coherente con la propuesta por $\mathrm{M}^{\mathrm{a}}$ Concepción Blasco que no descartaba (2007: 82) la aparición de las necrópolis de incineración a comienzos de este siglo. Aunque algunas de las dataciones absolutas podrían llevar hasta momentos más antiguos la aparición de las necrópolis de incineración, lo cierto es que no son totalmente concluyentes y los materiales arqueológicos recuperados no coinciden con los de los asentamientos más antiguos. Como en el caso de 
los asentamientos de la Edad del Hierro, las dataciones cronológicas no ayudan a precisar la cronología de un periodo por lo demás corto.

\subsection{Periodo de transición EHI - EHII}

La definición material y cronológica de este periodo es bastante compleja, ya que la existencia de dos grupos muy bien representados (Primera y Segunda edades del Hierro) deja la zona intermedia huérfana de otras variables que lo singularicen (fig. 8). Con todo, contamos con algunos datos de utilidad que nos pueden ayudar a identificar y reconstruir hasta cierto punto este proceso de la transición. La parte izquierda de esta zona del gráfico ya ha sido analizada dentro de la secuencia de la Primera Edad del Hierro y se situó cronológicamente en torno al comienzo del siglo $\mathrm{V}$ a.C. Hemos visto también cómo la introducción de técnicas constructivas que utilizan la piedra y el adobe no parecen justificar la existencia de una transición, pues ya se habían documentado en yacimientos de la Primera Edad del Hierro. Esta idea parece confirmarse ya que los yacimientos incluidos en este tramo, salvo el Cerro de las Nieves I, presentan estructuras realizadas en materiales no perecederos. Por el contrario, la presencia de cerámica a torno parece ser mucho más influyente en el proceso, ya que la mayoría de los yacimientos localizados a la derecha del gráfico - esto es, al final de este periodo de transición- se encuentran cercanos a la variable CeII, que aglutina yacimientos con porcentajes de cerámica a torno superiores al $50 \%$, mientras que los del comienzo del tramo se asociaban a porcentajes menores del 15\%. La separación entre los yacimientos del comienzo y del final del tramo es grande, algo que generalmente es un indicador de cambios rápidos (Jensen y Nielsen 1997a: 48), implicando por tanto una transición rápida de la Primera a la Segunda Edad del Hierro.

En cuanto a la definición cronológica, contamos con cuatro dataciones de TL y una de $\mathrm{C}_{14}$ que aportan información desigual. La única datación radiocarbónica, perteneciente a la fase I del Cerro de las Nieves (2640 \pm 110 bp. V.M. Fernández com. pers.) ofrece un resultado de 1080 - 440 a.C. calibrada a $2 \sigma$, con un intervalo demasiado amplio incluso sin valorar la posición del yacimiento en el AC, ya que la primera fase del poblado fue datada a través de los materiales recogidos a finales del siglo VI a.C. - siglo V a.C. (Fernández et al. 1994: 118-119). La datación podría ser explicada por la recogida de muestras de de vida muy larga -vigas de madera- para realizar el análisis.

Respecto de las dataciones por Termoluminiscencia, los resultados han sido muy desiguales. Para

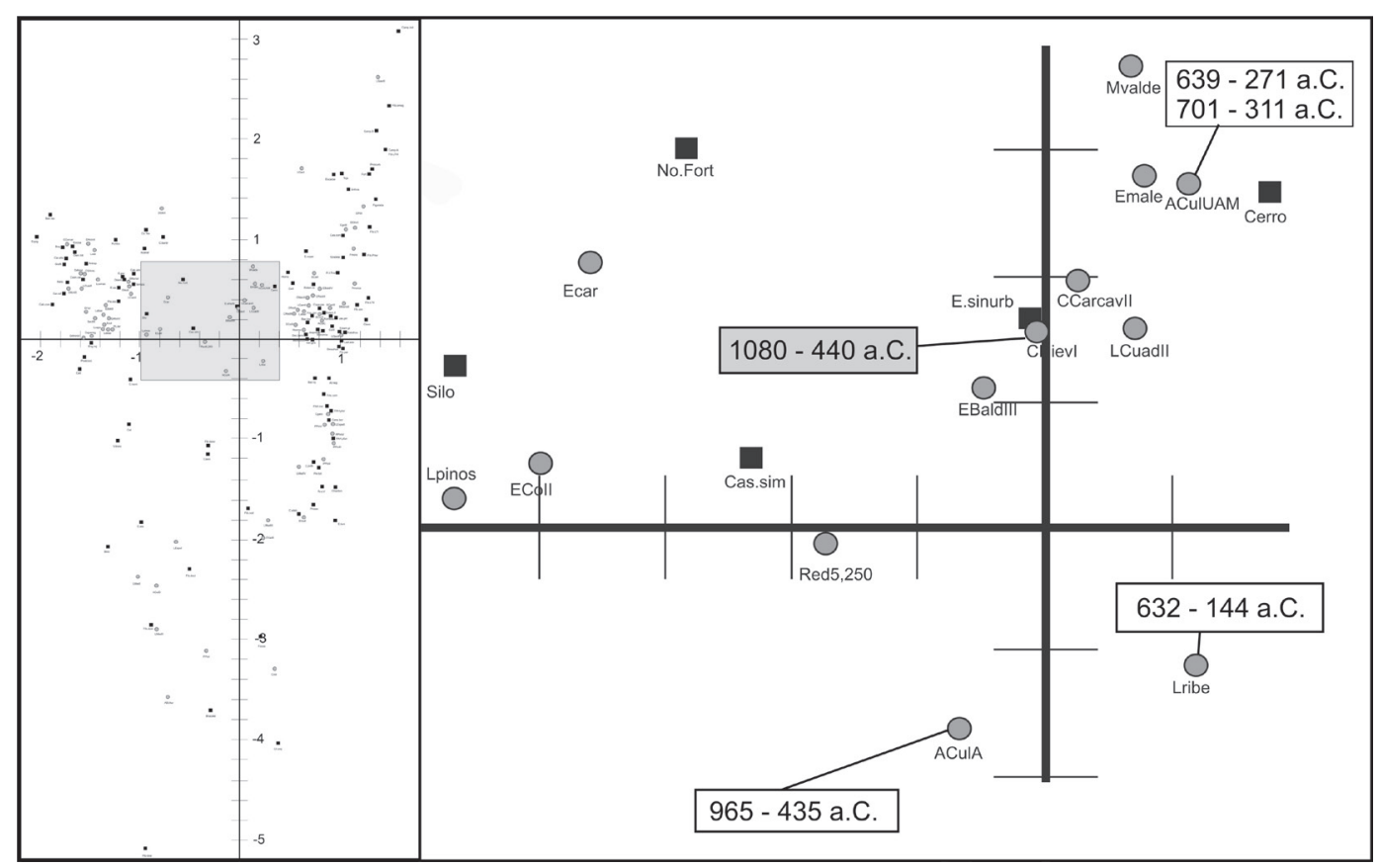

Fig. 8.- Localización de la zona de transición entre la Primera y la Segunda edades del Hierro en el gráfico general (izquierda) y ampliación de los yacimientos (círculos grises) y variables (cuadrados negros) con sus dataciones absolutas asociadas (derecha). Las dataciones radiocarbónicas (calibradas a $2 \sigma$ ) aparecen recuadradas en gris, las de termoluminiscencia, en blanco. 
Arroyo Culebro A (Penedo et al. 2002b: 309) se obtuvo una datación de $2650 \pm 265$, poco útil por su alto valor central y por su elevada desviación estándar. Otra de las dataciones que presenta algunos problemas es la fecha aportada por La Ribera (Galindo y Sánchez 2007: 288). Este yacimiento presenta un valor central es excesivamente bajo (siglo IV a.C.) y aunque la desviación es lo suficientemente amplia como para que pudiera localizarse en este tramo, su cultura material es más coherente con el periodo posterior y por tanto consideramos que su presencia en esta zona del gráfico constituye una anomalía.

Finalmente, las dos fechas obtenidas para el yacimiento de Arroyo Culebro excavado por la UAM son las que aportan mejores elementos de análisis (Rubio y Blasco 2000). Con unos intervalos de 639 - 271

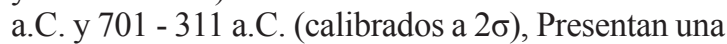
desviación estándar relativamente baja y unos valores centrales de 455 a.C. y 501 a.C. que se ajustan bien tanto a su posición dentro de la secuencia como a los indicios de cambios rápidos en la cultura material que se observan en el gráfico. Las fechas de Arroyo Culebro (UAM) podrían indicar por tanto el momento de consolidación del registro arqueológico tradicionalmente utilizado para caracterizar la Segunda Edad del Hierro, con presencia mayoritaria de cerámica a torno y construcciones en materiales no perecederos.

En el tránsito entre los siglos VI - V a.C. comienzan a detectarse las primeras cerámicas a torno en contextos de la Primera Edad del Hierro. La llegada de estas cerámicas no parece estar vinculada directamente con otro tipo de cambios en la cultura material -constructivos, metalúrgicos- que parecen haberse producido anteriormente y que no se generalizan de inmediato. La cerámica a torno, por el contrario, parece haberse difundido rápidamente, ya que a finales del siglo $\mathrm{V}$ a.C. representa más del $50 \%$ de la cerámica localizada en los yacimientos. Este salto podría ser explicado por la adquisición de la tecnología del torno, que facilitaría la producción y adquisición de este tipo de piezas. Si aceptamos que las pequeñas cantidades de cerámica a torno presentes en los yacimientos de principios del siglo $\mathrm{V}$ a.C. corresponden a importaciones o a los primeros intentos de fabricación, la adquisición de la tecnología del torno en la región debió producirse a mediados del siglo $\mathrm{V}$ a.C., expandiéndose en muy poco tiempo por todos los yacimientos.

Esta adquisición de la tecnología del torno no supone un cambio sustancial en las características de los asentamientos, que mantienen estructuras de habitación y patrones de asentamiento muy similares a los de siglos anteriores. Tan sólo un yacimiento -Cerro de las Nieves- presenta estructuras mucho más estables y porcentajes de cerámica a torno rela- tivamente altos. Sin embargo, el hecho de que este yacimiento se encuentre en una zona periférica de la región estudiada y en contacto más directo con influencias meridionales y mediterráneas puede justificar esta asimilación precoz de las nuevas modelos de asentamiento que se generalizarán posteriormente.

\subsection{Necrópolis de la Segunda Edad del Hierro}

Las necrópolis de la Segunda Edad del Hierro presentan una seriación muy clara (fig. 9), siguiendo quizá la tendencia más evidente de toda la secuencia. Esta coherencia es consecuencia directa de la calidad y la cantidad de información disponible. Contamos para este periodo con dos necrópolis con varias fases de ocupación (Las Madrigueras y $\mathrm{Pa}$ lomar de Pintado) de las que hemos accedido a la información completa bien a través de las memorias de excavación, bien a través del estudio directo de los materiales. Otra de las necrópolis (Cerro del Gato) fue documentada exhaustivamente durante la realización de nuestra tesis doctoral. Esta conjunción de necrópolis de larga duración e información abundante y bien caracterizada ha hecho que se haya logrado una seriación muy coherente y sin apenas anomalías, tanto en los yacimientos como en las variables. Cuando éstas aparecen, lo hacen en necrópolis como El Vado (Martín 2007; 2010), en las que la información disponible es menor. La calidad de la seriación se aprecia en cómo las fases de los diferentes yacimientos se distribuyen en un orden perfecto, de más antiguas a más modernas, al igual que las variables.

A través del gráfico, podríamos definir un momento en torno a siglo $\mathrm{V}$ a.C. asociado a las primeras cerámicas áticas en la región, vasos que imitan formas à chardon, cuchillos afalcatados y decoraciones de bandas que cubren toda la pieza. A estas necrópolis les suceden otras como Palomar de Pintado III y IV, datadas en el siglo IV-III a.C. y asociadas a la aparición de fíbulas anulares hispánicas de puente fundido y también a las primeras estructuras tumulares de la región. La fase final se dataría en torno a los siglos III - II a.C. y estaría representada por necrópolis como Palomar de Pintado V, Cerro del Gato y Las Esperillas II.

Por desgracia, este orden observado en el gráfico no está acompañado de un conjunto de dataciones absolutas bien ajustadas. Las nueve disponibles (seis radiocarbónicas y tres de termoluminiscencia), han sido realizadas en tumbas de Palomar de Pintado y presentan serios problemas de interpretación por su falta de adecuación a las fases arqueológicas propuestas, aunque los excavadores de la necrópolis encontraron algunos puntos de análisis 


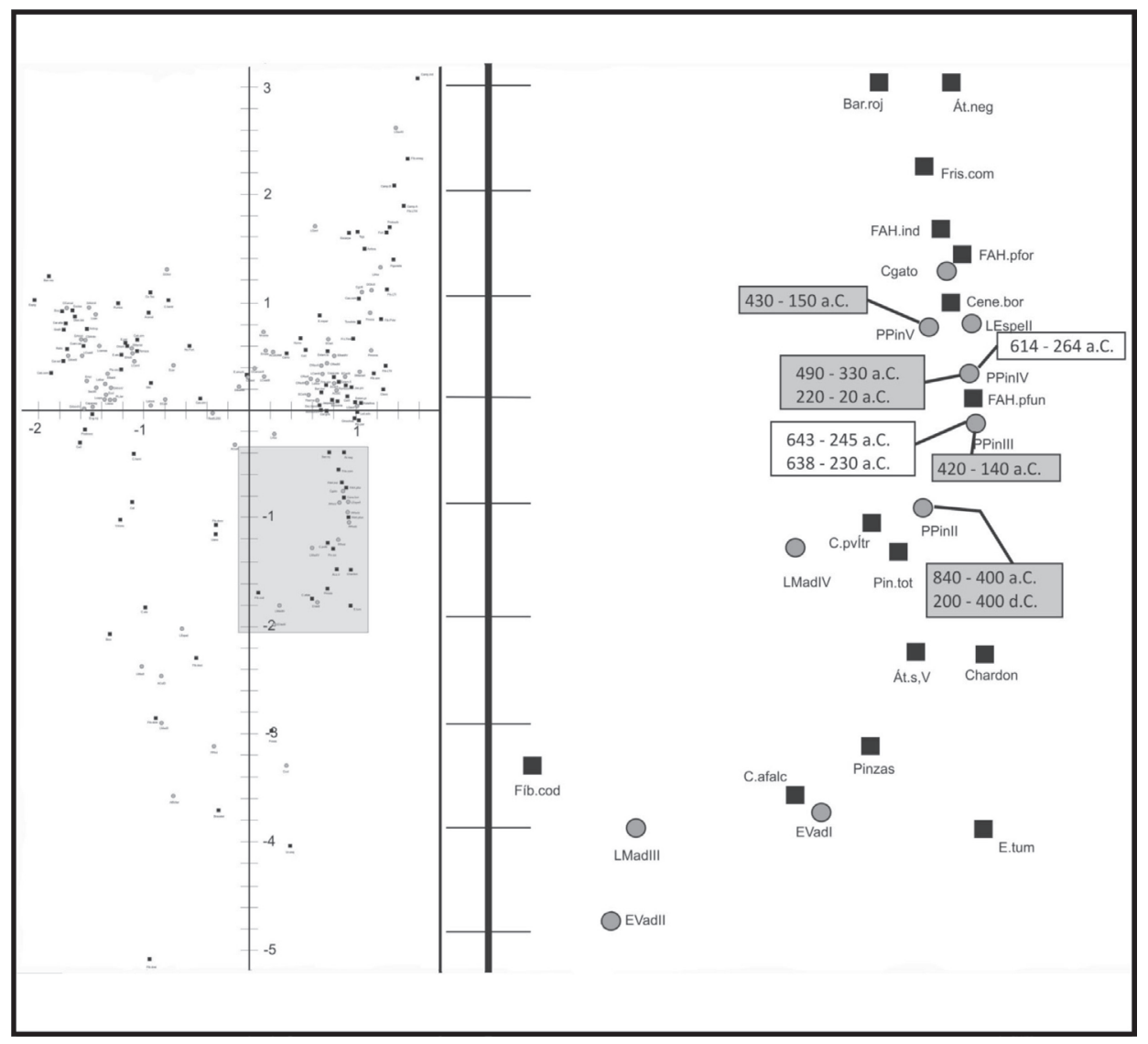

Fig. 9.- Localización de las necrópolis de la Segunda Edad del Hierro en el gráfico general (izquierda) y ampliación de los yacimientos (círculos grises) y variables (cuadrados negros) con sus dataciones absolutas asociadas (derecha). Las dataciones radiocarbónicas (calibradas a $2 \sigma$ ) aparecen recuadradas en gris, las de termoluminiscencia, en blanco.

útiles (Pereira et al. 2003). En las dataciones radiocarbónicas (fig. 9, cuadros con fondo gris), dejando de lado algunas dataciones claramente erróneas, los intervalos son demasiado amplios como para poder afinar en una cronología de tan sólo tres siglos. Cuando son un poco más ajustados, éstos no coinciden bien con las cronologías aceptadas para la cultura material.

En cuanto a las dataciones de termoluminiscencia (J. Pereira, com. pers.), las tres presentan fechas muy homogéneas con un valor centrado en torno al siglo IV a.C. (fig. 8 en blanco), lo que coincide bastante bien con las dos fases de Palomar de Pintado en las que se sitúan. Contamos además con una datación radiocarbónica y otra de termoluminiscencia para la misma tumba, la tumba 48 adscrita a la fase III de Palomar de Pintado. La fecha radiocarbónica es de 420 - 140 a.C. calibrada a $2 \sigma$ y la de TL 643 - 245 a.C., siendo el lapso común de ambas dataciones el siglo IV a.C. y la primera mitad del siglo III a.C. La cultura material de la fase en la que se encuadra la tumba 48 apunta a cronologías en torno a la primera mitad del siglo IV, ayudando a ajustar un horizonte arqueológico poco conocido ya que la fase datada corresponde al comienzo de los enterramientos tumulares en la zona de la mancha toledana/ conquense y que ha sido también documentada en necrópolis como El Vado (Martín 2010) y quizá también en Las Esperillas (García y Encinas 1987: 52).

Con estos resultados el estudio cronológico necesita apoyarse necesariamente en la cultura material. En este sentido, los indicadores son mucho más claros para las fases más antiguas, mientras que en la parte que corresponde a los siglos IV III a.C. los materiales, aun siguiendo un orden bastante lógico, aparece desplazados por la influencia estadística que ejercen los asentamientos donde 


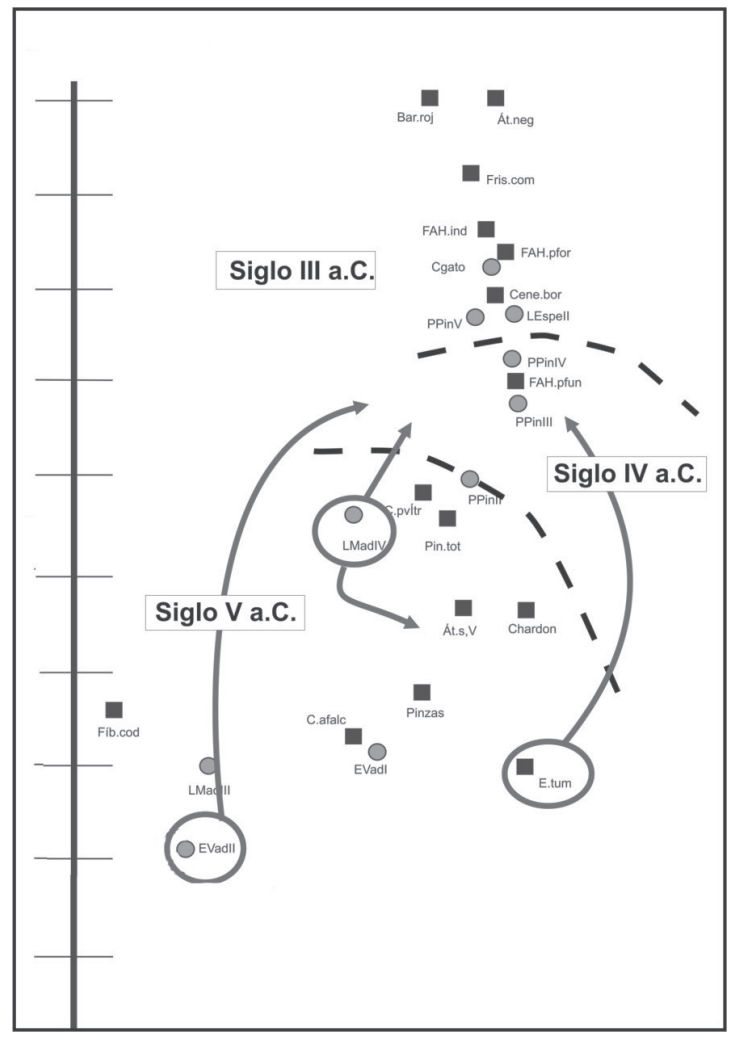

Fig. 10.- Interpretación cronológica de las necrópolis de la Segunda Edad del Hierro. Los cuadrados negros corresponden a variables, los círculos grises, a yacimientos. Las flechas indican la posición real de las anomalías detectadas.

también se han localizado. Es el caso sobre todo de la cerámica ática negra y la cerámica de barniz rojo, que aparecen tanto en asentamientos como en necrópolis y que en el gráfico se sitúan fuera del lugar que les correspondería si sólo se analizasen las necrópolis. Es un caso idéntico al analizado en el primer tramo de la secuencia respecto de las necrópolis de la Primera Edad del Hierro.

A partir de las cronologías ofrecidas por las necrópolis de la Primera Edad del Hierro, que podrían alcanzar los comienzos del siglo V a.C., parece haber un periodo poco claro de cambios bastante rápidos, que estaría representado solamente por Las Madrigueras III y que correspondería al periodo central del siglo $\mathrm{V}$ a.C. A finales de este siglo comienzan a generalizarse objetos de importación como las cuentas de pasta vítrea y las cerámicas áticas más antiguas, como se observa en El Vado I (Martín 2007: 262) y Las Madrigueras IV (Almagro 1969: 145).

En el siglo IV a.C., y al menos en la zona sudoriental del valle medio del Tajo, comienzan a aparecer los primeros enterramientos tumulares que conviven con las tumbas más tradicionales en forma de fosas circulares y más excepcionalmente, rectangulares o polilobuladas. La fase II de Palomar de Pintado, con materiales antiguos correspondería al momento previo (Ruiz et al. 2004: 125), y las fechas centrales de las dataciones de termoluminiscencia de Palomar de Pintado parecen apoyar esta cronología. Este periodo parece prolongarse hasta el siglo III a.C., y está representado por las fases III, IV y V de Palomar de Pintado (Ruiz et al. 2004: 126-7) y El Vado II. Se generalizan objetos que en fases anteriores eran escasos, como las cuentas de pasta vítrea, y aparecen las cerámicas áticas de barniz negro y las cerámicas de barniz rojo ibérico.

Poseemos pocos datos para valorar la fase final de las necrópolis de incineración desde finales del siglo III a.C. Palomar de Pintado V (en la zona oriental) conserva estructuras tumulares, pero es la única necrópolis de este momento que está bien descrita. Las Esperillas II aparece situada muy cerca de Palomar de Pintado V, y tenemos información de que se documentó al menos una estructura tumular (García y Encinas 1987: 52), pero la insuficiente información acerca de esta necrópolis hace que no podamos utilizar su posición en el gráfico como un apoyo firme para nuestra interpretación. Algo parecido ocurre con la necrópolis del Cerro del Gato, muy mal documentada aunque se hayan podido estudiar todos los materiales. Según comunicación personal de Salvador Llopis, todas las tumbas eran de fosa, sin que se documentaran otro tipo de estructuras. Esta ausencia podría deberse a una diferencia regional frente a las necrópolis de la zona sudoriental, o bien un rasgo que caracterizase la última etapa de las necrópolis de incineración. Por desgracia, carecemos de otros ejemplos para confirmar o rebatir esta propuesta.

Los principales problemas de inserción dentro de este esquema corresponden -además del caso ya descrito de las necrópolis del periodo final- a la necrópolis de Las Madrigueras, que como ya hemos dicho presenta algunas dudas en la adscripción cronológica de las tumbas a cada una de sus cuatro fases. En el caso de Las Madrigueras IV (la más moderna), las tumbas incluidas en la fase presentan un conjunto de materiales que corresponden tanto a finales del siglo $\mathrm{V}$ a.C. como al pleno siglo IV a.C., aunque Martín Almagro (1969: 145) sitúa esta fase a finales del siglo IV a.C. - principios del siglo III a.C. Hemos representado este problema indicando las zonas a las que correspondería Las Madrigueras IV según sus materiales a través de flechas, así como las otras anomalías detectadas en la distribución (la fase más antigua de El Vado y la variable que repre- 
senta a la aparición de estructuras tumulares, indicando su posición cronología correcta (fig. 10).

\subsection{La Segunda Edad del Hierro}

La zona del gráfico en la que se han representado el conjunto de yacimientos pertenecientes a la etapa plena de la Segunda Edad del Hierro (fig. 11) presenta una gran acumulación de asentamientos y variables. Esta acumulación nos habla de una etapa de estabilidad en la que los cambios son más lentos que en el periodo anterior, y que podría interpretarse como un periodo de plenitud en el que se consolidan las tendencias apreciadas en el momento de transición representado por el grupo 3 . Como en el conjunto correspondiente la Primera Edad del Hierro, se aprecia la influencia que ejerce el grupo de las necrópolis sobre la distribución de las variables de este periodo. En líneas generales la distribución del gráfico es bastante coherente con las cronologías propuestas por los materiales, aunque la acumulación de yacimientos y variables es tanta que es difícil delimitar una evolución cronológica ajustada para el tramo.
A simple vista puede apreciarse cómo las variables con cronologías más antiguas (estilos decorativos más simples, porcentajes de cerámica a torno más bajos) sitúan en la zona de la izquierda, mientras que aquellos con cronologías más modernas se sitúan más a la derecha, aunque algunos se encuentran desplazados hacia la parte inferior del gráfico por aparecer también en necrópolis. Del mismo modo, el gráfico parece confirmar la propuesta de Dionisio Urbina (2005) de la transformación de los patrones de asentamiento hacia un progresivo amurallamiento de los asentamientos. Los tres ejemplos de este tipo representados en el gráfico (La Gavia, Plaza de Moros y Santa María) se sitúan en la parte derecha del gráfico.

Las dataciones radiocarbónicas (figura 11, cuadros grises) no ayudan demasiado a establecer un horizonte preciso, algo hasta cierto punto lógico teniendo en cuenta la enorme concentración de asentamientos y variables en un periodo de tiempo no superior a dos siglos. Salvo excepciones pueden considerarse correctas, ya que engloban el periodo estudiado, pero con intervalos demasiado amplios como para ser de utilidad. Sí puede observarse un

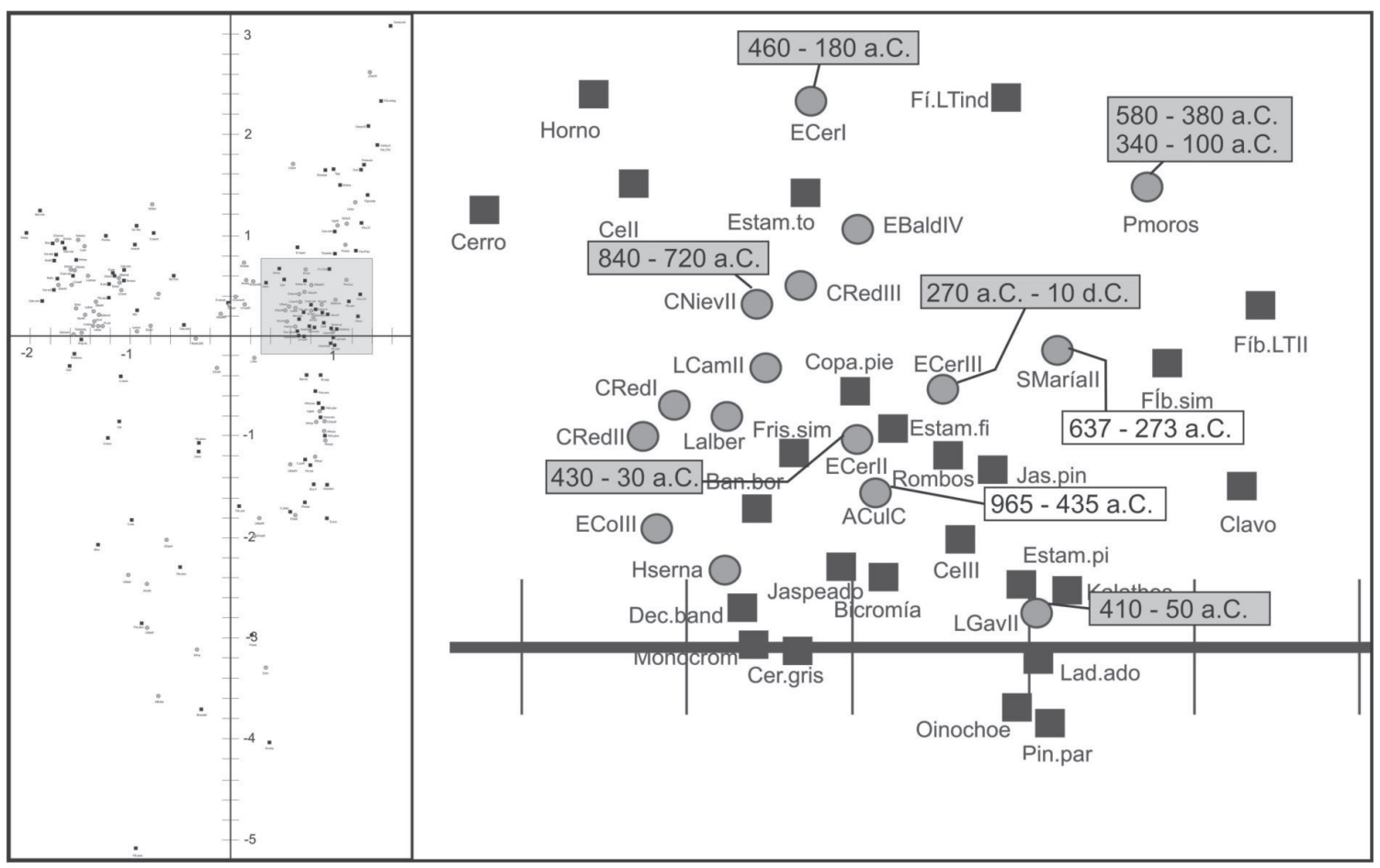

Fig. 11.- Localización de los asentamientos de la Segunda Edad del Hierro en el gráfico general (izquierda) y ampliación de los yacimientos (círculos grises) y variables (cuadrados negros) con sus dataciones absolutas asociadas (derecha). Las dataciones radiocarbónicas (calibradas a $2 \sigma$ ) aparecen recuadradas en gris, las de termoluminiscencia, en blanco. 


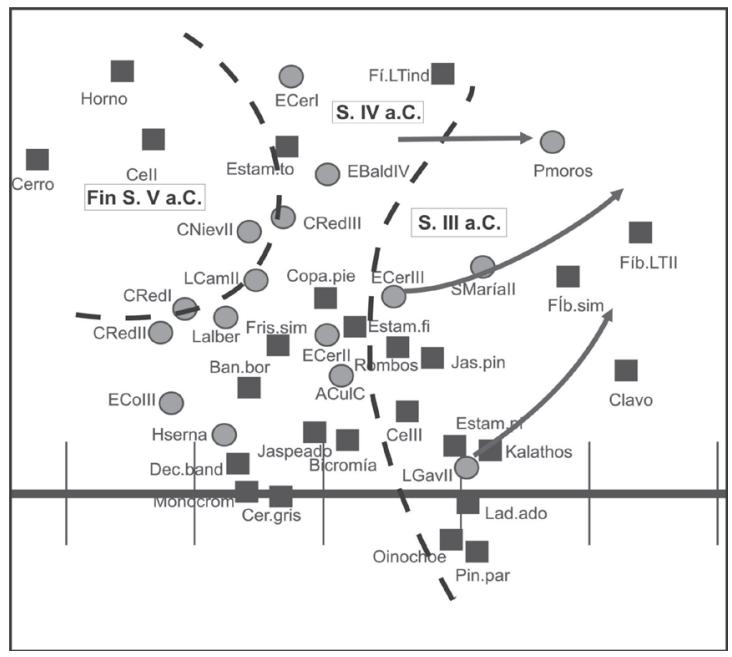

Fig. 12.- Interpretación cronológica de los asentamientos de la Segunda Edad del Hierro. Los cuadrados negros corresponden a variables, los círculos grises, a yacimientos. Las flechas indican la posición real de las principales anomalías detectadas.

ligero desplazamiento de las fechas, cuyo límite superior tiende a disminuir conforme nos acercamos al final del tramo. Por desgracia, una de las fechas más interesante, la de Plaza de Moros (Urbina y Urquijo 2004: 84), presenta una grave contradicción entre las dos dataciones tomadas de la misma muestra, lo que invalida en gran medida un resultado de 340 - 100 a.C. calibrado con CalPal a $2 \sigma$ que parece bastante ajustado.

Junto a esta datación, la obtenida para la tercera fase de El Cerrón (Valiente 1994: 330) también parece corresponder bastante bien (al menos, en su límite inferior) a las fechas que proporcionadas por los materiales. Otras dataciones son claramente incorrectas, como la obtenida para la segunda fase del Cerro de las Nieves $(2850 \pm 30$ bp, V.M. Fernández com. pers.), que pese a tener un intervalo muy ajustado adolece del mismo problema de excesiva antigüedad que ya discutimos para la datación de la fase I de este yacimiento en el grupo 3. Respecto de las dataciones por TL, la obtenida para Arroyo Culebro C (Penedo et al. 2002b: 309) es a todas luces muy alta, además de presentar las dudas ya establecidas en el capítulo correspondiente. La fecha de Santa María (Pérez y Bueno 2007: 338) es más aceptable, aunque un poco antigua para la parte del gráfico en que se sitúa. Con todos los problemas que conlleva la citada acumulación de yacimientos y variables, la interpretación del tramo de la secuencia puede establecerse tal y como se ve en la figura 12 .
El comienzo del periodo se dataría a finales del siglo V a.C. y principios del siglo IV a.C., representado por yacimientos como Cerro Redondo I, Las Nieves I, Hoyo de la Serna, Laguna del Campillo II y El Colegio III. Son yacimientos situados en llano o sobre suaves lomas, sin fortificaciones. La cerámica a torno es mayoritaria pero aún se documentan porcentajes significativos de piezas realizadas a mano, como ocurre desde mediados del siglo V a.C. El principal cambio respecto de la etapa anterior, considerada de transición, es la generalización de las construcciones realizadas con zócalos de piedra y recrecidos de adobe, o con adobes hechos a caja en el caso de Cerro Redondo (Blasco y Alonso 1985). La cultura material que viene asociada en el gráfico muestra tipos y estilos decorativos simples: decoración a bandas simples, frisos sencillos, monocromía, bandas pintadas en la parte interior del borde y aparición del jaspeado.

La mayoría de estos yacimientos prolongan $\mathrm{su}$ existencia durante todo el siglo IV a.C. detectándose en algunos de ellos (Cerro Redondo, El Cerrón) nuevas fases de ocupación y apareciendo algunos nuevos como Arroyo Culebro D. La cultura material no parece variar sustancialmente (aunque hay que tener en cuenta los problemas derivados de la distorsión ejercida en el gráfico por las necrópolis). Aunque aparecen alejados del resto, es el momento de la generalización de las cerámicas de barniz rojo y áticas negras.

A finales del siglo IV a.C. comenzaría a apreciarse un cambio en el patrón de asentamientos con la aparición de poblados en escarpes y zonas mejor defendidas. Este proceso continúa durante el siglo III a.C., en el que se aprecian cambios en la cultura material con la aparición o generalización visible en el gráfico de tipos como la copas de pie moldurado, los kalathos y oinochoe y nuevos estilos decorativos como la combinación de pintura y estampillado, los frisos complejos, los diseños de cenefas en el borde de las piezas o la combinación de pintura y estampillado. Los yacimientos más característicos de este periodo son, como hemos dicho, los fortificados como Plaza de Moros, La Gavia o Santa María, aunque otros asentamientos en llano parecen haber seguido existiendo, como El Cerrón III. En este sentido, hay que aclarar que AC sitúa a los yacimientos por criterios de mayor o menor cercanía, pero no puede representar la perduración del yacimiento a lo largo del tiempo (a no ser que se disponga de información sobre varias fases). Así, El Cerrón III se ha situado en un punto en torno a finales del siglo IV a.C. - principios del siglo III a.C. a partir de las características de su cultura material, aunque su perduración cronológica probablemente abarca todo el siglo III a.C. Del mismo modo, se ha 
defendido una cronología para Plaza de Moros de los siglos IV - III a.C. En el gráfico aparece situado algo alejado, cerca de variables asociadas al siglo III a.C. Lo importante es la percepción del cambio de modelo, que por otra parte no es inmediata. La conclusión a extraer es que yacimientos como $\mathrm{El}$ Cerrón son más característicos de etapas más antiguas, aunque sigan existiendo en siglos posteriores, mientras que yacimientos como Plaza de Moros podrían haber comenzado a aparecer en el siglo IV a.C., pero cuando se hacen verdaderamente representativos del poblamiento de la región es en el siglo III a.C. Esta situación se ha representado en la figura 12 con flechas que indican de dónde o hacia dónde se prolongan temporalmente algunos de los yacimientos más representativos

En la parte final del tramo (a finales del siglo III a.C.) se observa una progresiva separación de variables y yacimientos, comparada con la agrupación anterior. Esto simboliza, como hemos visto en varias ocasiones, el inicio de cambios rápidos en la cultura material. No es éste el lugar para contextualizar históricamente estos cambios, pero es significativo que coincidan cronológicamente con el comienzo de la presencia cartaginesa y poco después romana en la región. Estos cambios rápidos, como vamos a ver a continuación, van a acentuarse en el tramo final de la secuencia, durante el periodo de conquista y control romano.

\subsection{El final de la Edad del Hierro}

Junto con el grupo que representa a las necrópolis de la Segunda Edad del Hierro, esta fase final (fig. 13) es quizá la que mejor reproduce las características de la parábola tipo a la que idealmente deben tender los AC cuando están representando una seriación. El número de yacimientos y variables es bastante escaso, y se encuentran con alguna excepción en un orden cronológico muy coherente. Todos los yacimientos situados en este grupo han sido siempre considerados de cronología tardía, entre los siglos II-I a.C. Este hecho no quiere decir que no puedan haber existido en etapas anteriores, simplemente indica cuál es el momento en que este tipo de asentamientos son más representativos.

Las dataciones radiocarbónicas del periodo (en recuadros grises en la figura 13) son muy coherentes con la cronología que muestran los materiales y con la ordenación general del tramo. Contamos con un magnífica serie de dataciones del Llano de la Horca (Märtens et al. 2009: 211-12), que apuntan a que es a partir del siglo II a.C. cuando parece producirse la máxima expansión del poblamiento en el cerro y éste es el periodo al que pertenecen los materiales recogidos en el yacimiento. Esta conclusión es coherente con La Gavia III, que presenta la datación

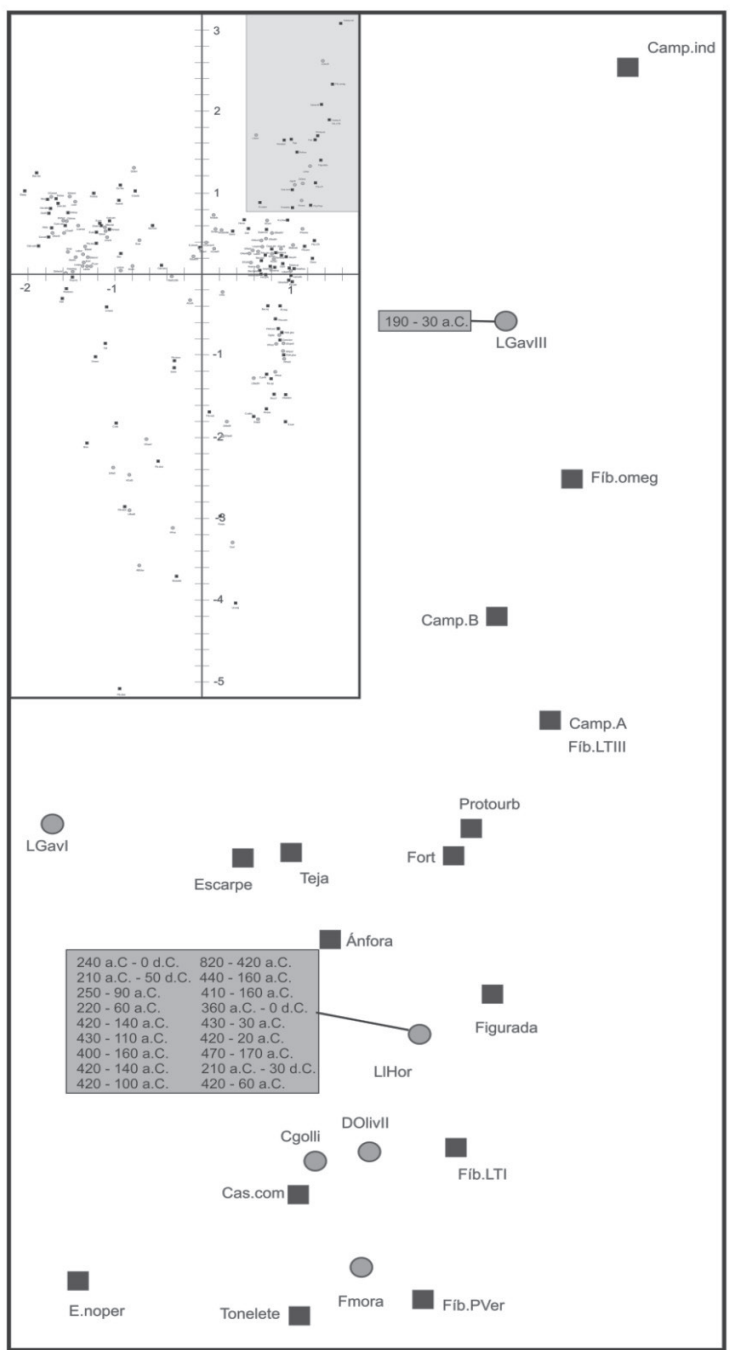

Fig. 13.- Localización de los asentamientos del final de la Segunda Edad del Hierro en el gráfico general (arriba a la izquierda) y ampliación de los yacimientos (círculos grises) y variables (cuadrados negros) con sus dataciones absolutas asociadas (derecha). Todas las dataciones (recuadradas en gris) son radiocarbónicas calibradas a $2 \sigma$.

más moderna de la serie y cuya fase corresponde a un periodo de ocupación marginal del cerro (Quero et al. 2005: 139). Así pues, las dataciones muestran un horizonte totalmente coherente con la cultura material de los yacimientos en torno a los siglos II I a.C., añadiendo una ocupación anterior desde los siglos IV - III a.C. para el Llano de la Horca. En el caso del yacimiento de la Dehesa de la Oliva, el numerario recogido señala claramente un horizonte de los siglos II-I a.C. (Cuadrado 1991: 231, Montero y Sejas 2003-2004). 
Con estos datos pueden proponerse, grosso modo, dos momentos relevantes en este tramo. En torno al siglo II a.C. se produce la aparición o crecimiento de un tipo de yacimientos diferentes a los de épocas anteriores: grandes, fortificados y con las primeras evidencias de urbanismo. Aunque el proceso es poco conocido, es probable que estos yacimientos existieran en etapas anteriores -algo que parece claro, por lo menos, para el Llano de la Horca y La Gavia. La cultura material es similar a la anterior, con presencia de materiales muy característicos del periodo como las fíbulas de La Tène y omega o las cerámicas de decoración figurada, junto a las primeras muestras de presencia romana (bien como elementos de importación, bien traídos por las tropas en su conquista de la región).

El siglo I a.C. marca el fin de la secuencia y de la Edad del Hierro en la región. El único yacimiento claramente adscrito a este periodo es La Gavia III, con una ocupación residual del cerro, mientras que otros yacimientos como el Cerro del Gollino (Santos et al. 1998) o el Llano de la Horca (Märtens et al. 2009) quedan desocupados. Las causas pueden ser varias: comienzo real de la romanización en la región tras un periodo de simple ocupación militar, reestructuración del sistema de ocupación del territorio, reacciones asociadas a las guerras sertorianas, o más bien una mezcla de todos estos factores. En cualquier caso, parece que el final de la Segunda Edad del Hierro debe situarse más cercano al cambio de era que al momento de la conquista romana a principios del siglo II a.C.

\section{Conclusiones}

Aunque presentado de manera muy breve, consideramos que el análisis de correspondencias realizado ha conseguido construir satisfactoriamente una seriación de la evolución de asentamientos y sus variables asociadas a lo largo de la Edad del Hierro en el valle medio del Tajo, integrando de manera visualmente muy expresiva y coherente la práctica totalidad de yacimientos conocidos para este periodo. La combinación del $\mathrm{AC}$ con el conjunto de dataciones absolutas -pese a todos los problemas que éstas presentan- ha permitido ofrecer, por primera vez, una secuencia cronológica general del primer milenio a.C. en nuestra zona. Esta secuencia es coherente con las propuestas cronológicas previas realizadas para momentos más específicos de la Edad del Hierro, pero además las unifica y refuerza ya que aporta el plus de objetividad del análisis estadístico, además de afinar algunas las cronologías que hasta ahora estaban poco definidas.

Más aún, las características del $\mathrm{AC}$ han permitido situar cronológicamente algunos yacimientos mal defi- nidos materialmente, agrupándolos junto a otros mejor conocidos, facilitando de este modo su comparación y sentando las bases para un análisis conjunto de su cultura material. Asimismo, ha generado información sobre el ritmo y secuencia de algunos procesos poco conocidos, como la transición entre la Primera y Segunda Edades del Hierro o sobre la existencia de dos momentos cronológicos en las necrópolis de incineración de la Primera Edad del Hierro. Los resultados del análisis han sido sintetizados en la figura 14 , donde se ha ordenado cronológicamente el primer milenio a.C. en el valle medio del Tajo a través de sus asentamientos y necrópolis mejor conocidas y de la cultura material más significativa localizada en los yacimientos. Esta síntesis no debería tomarse como un cuadro cronotipológico al uso, ni pretende serlo. Ha sido realizada a partir de los resultados de un análisis estadístico, y por tanto muestra tendencias o agrupaciones generales De este modo, aunque los yacimientos y variables asociadas presentan cierto orden cronológico, éste no es absoluto, y aquellos que se encuentran dentro de un mismo recuadro comparten generalmente muchos rasgos comunes.

Pese a su imperfección, consideramos que el cuadro resume bastante bien las principales transformaciones de la cultura material a lo largo del primer milenio a.C. en la región, y puede resultar útil para mejorar progresivamente la adscripción cronológica de nuevos yacimientos excavados, ayudando a establecer una secuencia definitiva para la Protohistoria del valle medio del Tajo.

Por otra parte, aunque el Análisis de Correspondencias aquí presentado tiene una interpretación eminentemente cronológica, algunos de los elementos analizados en el artículo tienen unas connotaciones que superan la simple asociación yacimientos-cultura material. Es el caso de la aparición de las necrópolis de incineración en un momento relativamente avanzado de la Edad del Hierro, tiempo después de la sedentarización definitiva de la población de la región, lo que podría indicar un proceso de adaptación más lento en la esfera ideológica que en la económica. Del mismo modo, los resultados del AC matizan la idea de un cambio tecnológico global -torno, siderurgia, construcciones en materiales no perecederos- que marcara la transición a la Segunda Edad del Hierro. Esta transición -si existe- no parece estar asociada tanto a la introducción de nuevas tecnologías como a posibles cambios dentro de las comunidades que habitaron el valle medio del Tajo.

Finalmente, es necesario valorar hasta qué punto las transformaciones que observamos en el AC tienen correspondencia en otro tipo de ámbitos que no han sido representados en este análisis (económico, demográfico, social, etc.). En este sentido, el análisis del resto de variables (patrones de poblamiento, datos económicos, modelos de organización social) muestra diferencias 


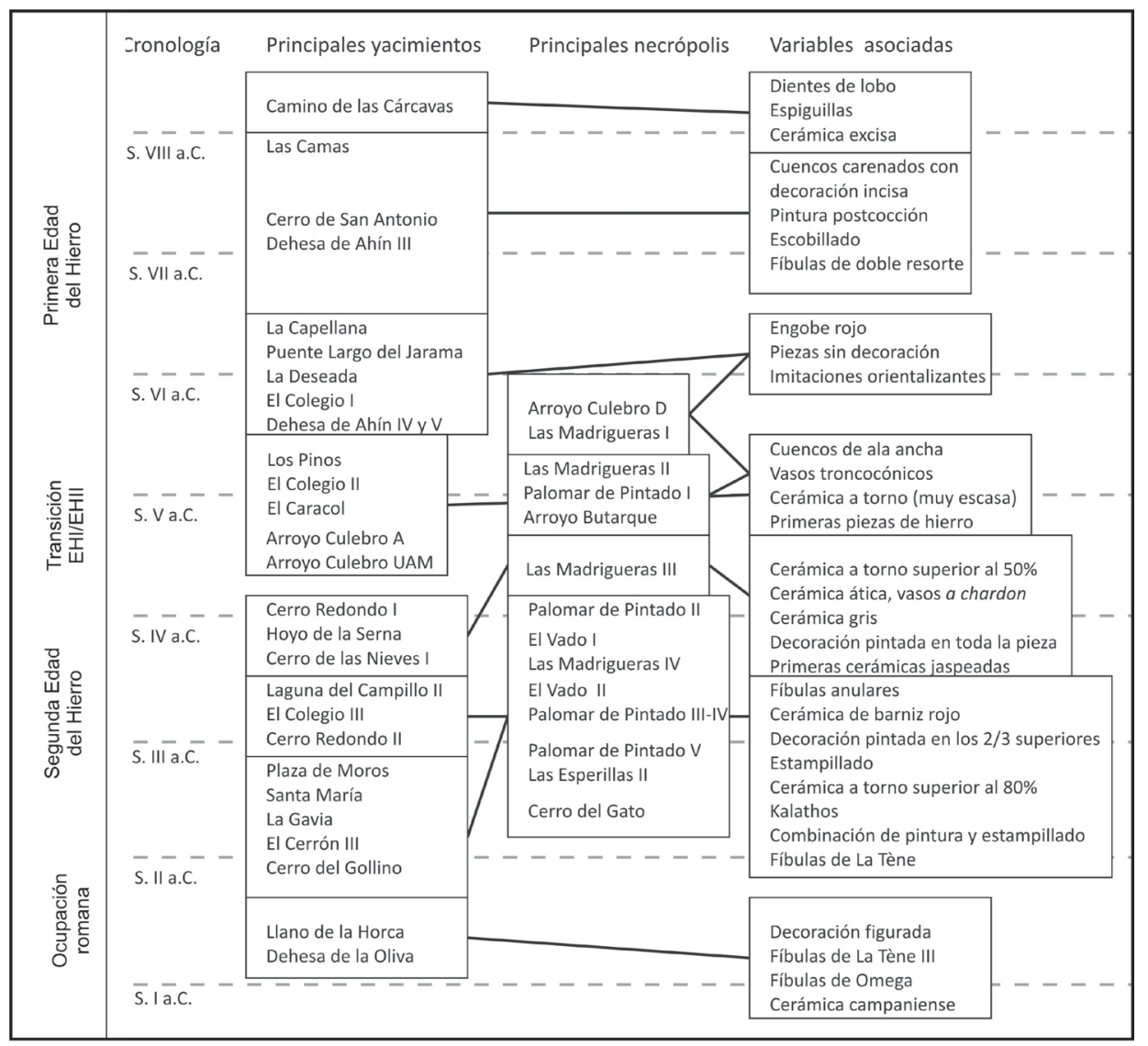

Fig. 14.- Interpretación de la seriación obtenida a través del análisis de correspondencias, representando los principales yacimientos junto a las variables más significativas a las que aparecen asociados.

sorprendentes con la disposición de nuestras variables y el resto de información disponible. Así, todos los datos conocidos apuntan a una enorme estabilidad en el poblamiento de la región desde el comienzo de la Edad del Hierro hasta el siglo IV a.C. (Torres 2012: 418), con un mantenimiento de los patrones de ocupación del territorio y del tipo de hábitat, disperso y sin fortificaciones. Sin embargo, en ese mismo periodo el AC muestra cambios sustanciales en la cultura material, incluida la sustitución de construcciones perecederas por otras realizadas en piedra, la introducción del torno o la llegada de importaciones procedentes del ámbito mediterráneo. En el sentido contrario, el AC muestra una enorme estabilidad en el tramo correspondiente a la Segunda Edad del Hierro, mientras que es en ese momento cuando se produce uno de los cambios más importantes en la región: la aparición de asentamientos amurallados y la remodelación de los patrones de asentamiento de la región (Urbina 2005). Aunque el AC recoge adecuadamente la posición de este tipo de yacimientos, su distribución rechaza la existencia de cambios bruscos a lo largo del tiempo en la cultura material.

Estos dos ejemplos llevan a una reflexión interesante sobre el grado en que los cambios materiales tienen su correspondencia en otro tipo de transformaciones. Un análisis contrastado de los resultados de nuestro $\mathrm{AC}$ respecto de otras variables muestra que ambos no siempre van de la mano, abriendo la puerta a otro tipo de interpretaciones de tipo socioeconómico. Dado el enfoque de nuestra trabajo, las posibilidades de análisis de este tipo de procesos es muy limitada, aunque la posibilidad de detectar patrones de organización social a través de AC está suficientemente contrastada (Jensen y Nielsen 1997b) y ya ha sido aplicada en el valle medio del Tajo con buenos resultados (Torres y Penedo 2009).

En este sentido, los últimos análisis de la estructura social de las comunidades del valle medio del Tajo (Torres 2012) apuntan a un alto grado de igualdad social durante la Edad del Hierro. Los primeros cambios son perceptibles exclusivamente en el contexto fune- 
rario a partir del siglo VI a.C., asociados a procesos de competición entre grupos familiares (Torres y Penedo 2009: 371), algo que podría explicar la separación de las necrópolis del resto de yacimientos. Esta situación parece mantenerse a lo largo del siglo V a.C., reforzando la idea de que los cambios materiales detectados en el AC no tienen una correlación directa con las transformaciones sociales. A mediados del siglo IV a.C., la -relativa- igualdad social parece estallar de manera diferente en el núcleo central del valle y en la región del sudeste. En esta región, las diferencias socioeconómicas se hacen cada vez más evidentes especialmente en el mundo funerario, con la aparición de estructuras tumulares que imitan las documentadas en la región de Albacete (Blázquez 1999). En el caso del centro del valle, la expresión de las tensiones sociales devino en una conflictividad mucho más explícita que modificó los patrones de poblamiento obligando a fortificar los asentamientos en un clima de creciente tensión. El cambio definitivo, sin embargo, no se produjo hasta la conquista romana a comienzos del siglo II a.C., cuando comienzan a modificarse todos los ámbitos de la vida de estas poblaciones.

Por supuesto, un análisis tan complejo como el presentado y con un conjunto de datos tan variable no está exento de contradicciones en la distribución. Aunque en la discusión de los resultados se han obviado por reiterativas, las principales anomalías en el análisis están asociadas a tres tipos de problemas. El más común es la falta de datos suficientes, especialmente cuando un yacimiento aparece definido por menos de cinco variables o una variable no aparece al menos en cinco yacimientos. Las diferencias entre la cultura material de necrópolis y asentamientos han afectado a la uniformidad de la secuencia, dificultando la correcta localización de algunas variables. Finalmente, uno de los conjuntos de variables seleccionadas inicialmente para el análisis ha demostrado tener escasa o ninguna utilidad para estructurar la secuencia. Es el caso de las relacionadas con las características estructurales, topográficas y constructivas de los yacimientos, que en general no han aportado información novedosa o relevante que ayude a definir los diferentes periodos de la Edad del Hierro. Todas estas anomalías señalan claramente cuál sería la situación ideal para realizar un nuevo Análisis de Correspondencias: centrado exclusivamente en la cultura material de los yacimientos, con un número mínimo de 5 - 10 variables y realizado por separado en yacimientos y necrópolis. Si seguimos estas pautas, la forma de la parábola es casi perfecta, como puede apreciarse en la figura 15, aunque la disposición de asentamientos y variables no varía significativamente.

Aunque lejos de ser perfecto, el AC aquí presentado ha demostrado ser una herramienta útil para

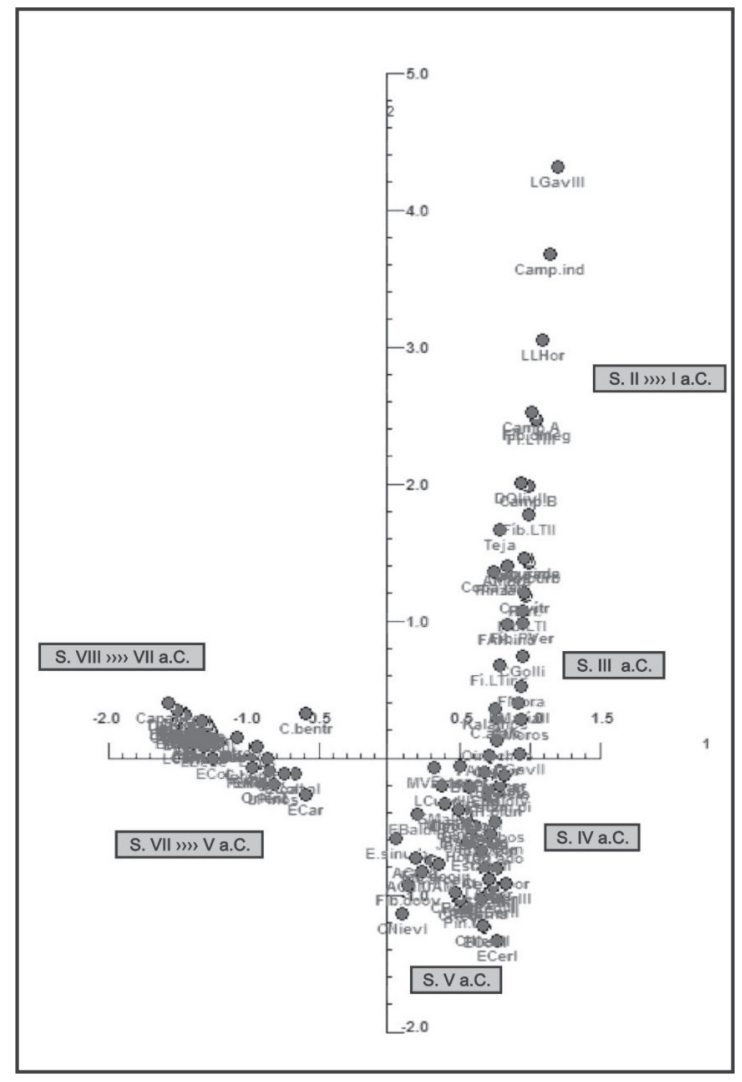

Fig. 15.- Análisis de correspondencias optimizado tras las correcciones de los fallos detectados en el análisis inicial.

afrontar la gestión e interpretación de conjuntos de datos cualitativos de valor y cantidad muy desiguales, en este caso, en una secuencia para este caso de más de ocho siglos de duración. Su aplicación ha permitido sortear satisfactoriamente algunas dificultades recurrentes en arqueología, como las derivadas de la publicación parcial de los datos, por lo que esta metodología podría ser utilizada en otras zonas y cronologías que presenten problemas similares. Aunque como todos los análisis estadísticos simplemente apunta tendencias, abre un camino para integrar datos difícilmente manejables desde otras posturas metodológicas más clásicas y para realizar análisis más complejos una vez que se ha conseguido el paso inicial de obtener la secuencia cronológica básica. Como cualquier análisis estadístico, no sólo debería servir para presentar e interpretar los resultados materiales, sino que debería abrir la puerta a nuevos análisis que mejoren, depuren y amplíen el modelo $\mathrm{y}$ que sienten las bases para su interpretación dentro de un discurso histórico que hasta ahora ha sido poco explorado en la historiografía de nuestra región ${ }^{1}$. 


\section{Apéndice}

Variables y yacimientos utilizadas en el análisis de correspondencias ${ }^{2}$

Acanal decoración acanalada. Ánfora. Antrop (antropomorfo). Representación de seres humanos a través de cualquier técnica (pintura, incisión, etc.) sobre cerámica. At.neg. Cerámica ática datada en pleno siglo IV. At.sV Cerámica ática datada entre finales del siglo $\mathrm{V}$ a.C. y principios del siglo IV a.C. Ban.bor (banda plana en borde). Banda de pintura que recubre la parte interna superior del borde de una pieza. Ban.res presencia de decoración en zigzag con bandas reservadas. Bar.roj Cerámica de barniz rojo. Bico pieza de perfil bicónico. Bicromía Boq Boquique. Brazalet Brazalete de bronce Cab.com (Cabañas complejas). Estructuras de habitación utilizadas como vivienda, realizadas en materiales perecederos y con espacio interno está compartimentado con ambientes separados para diferentes actividades. Cab.sim (Cabañas simples). Estructuras de habitación realizadas en materiales perecederos y con un espacio interno diáfano C.afalc Cuchillo afalcatado C.ala Cuencos de borde vuelto plano o ala. Camp.A Cerámica campaniense tipo A. Camp.B Cerámica campaniense tipo B. Camp.ind Cerámica campaniense indeterminada. Car.alta: Vasijas con carena alta. Car.esf Piezas con carena alta y cuerpo esférico. Cas.Com (Casas complejas). Estructuras de habitación utilizadas como vivienda realizadas en materiales no perecederos con diferenciación de los espacios funcionales en el interior. Cas.sim (Casas simples): estructuras de habitación utilizadas como vivienda realizadas en materiales no perecederos con espacio interno diáfano. C.bentr Cuencos de borde entrante y forma globular. Ce0 Proporción del 100\% de cerámica a mano. CeI Porcentaje de cerámica a torno hasta el $15 \%$. CeII. Porcentaje de cerámica a torno entre el 50 y el $80 \%$. CeIII Porcentaje de cerámica a torno superior al $80 \%$. Cene.bor (cenefas en borde). Borde de pieza pintado con algún tipo de cenefa: triángulos, ondas, trazos simples, etc. Cer.gris Cerámica gris ibérica Cerro yacimiento situado sobre una elevación suave, cerro o loma Cer.Tes Cerro testigo Chardon piezas a chardòn o que imitan esta forma, C.hemi (cuenco hemiesférico). Copa.pie copas de pie alto y generalmente moldurado. C.pvítr Cuentas de pasta vítrea. Cuen.car (cuencos carenados). Cuencos carenados con decoración incisa sobre la carena. Dec.ban decoración simple a bandas o líneas horizontales. Dien. lob decoración tipo "dientes de lobo". E.ais (Edificio aislado): Estructura aislada o asociada a otras estructuras de producción (pero no de habitación). E.noper Edificios construidos con materiales no perecederos En.roj Engobe rojo E.per Edificios construidos con materiales perecederos Escarpe Yacimiento situado sobre un escarpe. E.sinurb Estructuras sin urbanismo que pueden compartir infraestructuras comunes pero sin elementos de organización espacial como calles o muros. Espig uso de motivos formando espigas. Estam.ib Estampillados realizados en piezas de tipo ibérico, que no presenten decoración pintada. Estam. pi Combinación de pintura y estampillado, en piezas de cerámica de tipo ibérico. Estam.to Estampillados realizados en piezas toscas de almacenamiento tipo dolia o cerámicas destinadas a la cocina E.Tum Estructuras tumulares. Excisa decoración excisa FAH. indet Fíbula anular hispánica indeterminada. FAH. pfor Fíbula anular hispánica de puente forjado. FAH. pfua Fíbula anular hispánica de puente fundido al anillo. FAH.pfun Fíbula anular hispánica de puente fundido. Fíb.cod Fíbula de codo. Fíb.doci Fíbula de doble resorte con puente de cinta. Fíb.doin Fíbula de doble resorte indeterminada. Fíb.doov Fíbula de doble resorte con puente oval o circular. Fíb.dosi Fíbula de doble resorte con puente de sección simple. Fíb. LTI Fíbula La Tène con pie libre. Fíb.LTII Fíbula La Tène con pie sujeto. Fíb.omeg Fíbula de omega Fíb. PVer Fíbula de pie vertical. Fíb.sim Fíbula simétrica. Fí.LTIII Fíbula La Tène con pie fundido. Fí.LTind Fíbula La Tène indeterminada. Figurada Decoración pintada figurativa. Fort Asentamiento fortificado. Fosas necrópolis caracterizadas por fosas excavadas en el suelo o en la roca. Fris.com decoración pintada de frisos complejos combinando bandas y líneas con más de dos elementos decorativos. Fris.sim decoración de frisos simples combinando bandas y otros elementos decorativos. Grafit Grafitado. Horno Horno de ámbito supradoméstico. Jaspeado Decoración jaspeada Jasp.pin Combinación de jaspeado y pintura Kalathos. Lad.ado Ladrillos de adobe cocido hechos con caja. Llano yacimiento situado en llano o en ladera suave. Monocro Monocromía No.Fort Asentamiento sin fortificar Oinochoe. Oinochoe o jarra trilobulada. Orient Elementos orientalizantes o de inspiración orientalizante. Protourb (Protourbanismo). Agrupación de estructuras de habitación y producción organizadas a partir de elementos de distribución espacial (calles, plazas, muros) que estructuran el espacio del asentamiento. Pie.esc piezas con decoración escobillada Pint.par Piezas parcialmente pintadas (generalmente los dos tercios superiores). Pint.tot (Pieza totalmente pintada). La pintura o el jaspeado se distribuyen a lo largo de toda la pieza (no es necesario que la cubra totalmente). Pinzas Pinzas de bronce. Postcoce Pintura postcocción Puntos decoración puntillada. Retic Retículas incisas muy profundas y marcadas. Rombos. Rombos y otros diseños rectangulares pintados. Silos. Terraza: yacimiento situado en terraza. Tonelete. Ur.orej. Urna de orejetas. V.tronc Vaso troncocónico. 


\begin{tabular}{|c|c|c|}
\hline Código de yacimiento & Fases & Bibliografía utilizada en el análisis \\
\hline AButar (Arroyo Butarque) & AButar (EHI) & (Blasco et al. 2007) \\
\hline ACulA (Arroyo Culebro A) & ACulA (Transición EHI-EHII) & (Penedo et al. 2002a) \\
\hline ACulC (Arroyo Culebro C) & ACulC (EHII) & (Penedo et al. 2002a) \\
\hline ACuID (Arroyo Culebro D) & ACulD (EHI) & (Penedo et al. 2002c) \\
\hline $\begin{array}{l}\text { ACulUAM (Arroyo Culebro } \\
\text { UAM) }\end{array}$ & ACulUAM (Transición EHI-EHII) & (Blasco et al. 1998) \\
\hline Capaneg (Capanegra) & Capaneg (EHI) & (Martín y Virseda 2005; Martín 2007a) \\
\hline CCarcav (Camino de las Cárcavas) & CCarcavI (EHI) CCarcavII (EHII) & (López et al. 1999; Ortiz et al. 2007) \\
\hline CCol (Cerro Colorado) & CCol (EHII) & (Urbina y Urquijo 2007) \\
\hline CGato (Cerro del Gato) & CGato (EHII) & (Llopis 1950a; 1950b) \\
\hline CGolli (Cerro del Gollino) & CGolli (EHII) & (Santos et al. 1998) \\
\hline CNiev (Cerro de las Nieves) & $\begin{array}{|lll|}\begin{array}{l}\text { CNievI (Transición EHI-EHII) CNievII } \\
\text { (EHII) }\end{array} & \\
\end{array}$ & $\begin{array}{l}\text { (Fernández 1988; Fernández y Hornero 1988; } \\
\text { Fernández et al. 1994) }\end{array}$ \\
\hline CR (Cerro Redondo) & CRI (EHII) CRII (EHII) CRIII (EHII) & (Blasco y Alonso 1985) \\
\hline CSAnto (Cerro de San Antonio) & CSAnto (EHI) & (Blasco et al. 1991) \\
\hline DAhín (Dehesa de Ahín) & $\begin{array}{l}\text { DAhínI (EHI) DAhínII (EHI) DAhínIII } \\
\text { (EHI) DAhínIV (EHI) } \\
\text { DAhínV (EHI) } \\
\end{array}$ & (Rojas et al. 2007) \\
\hline DOliv (Dehesa de la Oliva) & DOlivI (EHI) DOlivII (EHII) & (Cuadrado 1991; Montero y Sejas 2003-2004) \\
\hline EBald (El Baldío) & $\begin{array}{l}\text { EBaldI (EHI) EBaldIII (EHII) } \\
\text { EBaldIV (EHII) }\end{array}$ & (Martín y Walid 2007) \\
\hline ECar (El Caracol) & ECar (Transición EHI-EHII) & (Oñate et al. 2007) \\
\hline ECer (El Cerrón) & $\begin{array}{|lllll|}\text { ECerI } & \text { (EHII) } & \text { ECerII } & \text { (EHII) } & \text { ECerIII } \\
\text { (EHII) } & & & & \\
\end{array}$ & (Miguel y Morales 1994) \\
\hline ECol (El Colegio) & $\begin{array}{l}\text { ECol (EHI) EColII (Transición EHI-EHII) } \\
\text { EColIII (EHII) } \\
\end{array}$ & (Sanguino et al. 2007a; 2009) \\
\hline EHo (Ecce Homo) & EHoI (EHI) EHoII (EHII) & (Almagro 1976; Almagro y Dávila 1988) \\
\hline EMale (El Malecón) & EMale (EHII) & (Rodríguez 2007) \\
\hline EVad (El Vado) & EVadI (EHII) EVadII (EHII) & (Martín 2007b, 2010) \\
\hline FMora (Fuente de la Mora) & FMora (EHII) & (Vega et al. 2009) \\
\hline HSerna (Hoyo de la Serna) & HSerna (EHII) & (Urbina et al. 2001) \\
\hline LAlbar (La Albareja) & LAlbar (EHI) & (Consuegra y Díaz del Río 2007) \\
\hline LAlber (La Alberquilla) & LAlber (EHII) & (Gutiérrez et al. 2007) \\
\hline LCam (Laguna del Campillo) & LCamI (EHI) LCamII (EHII) & (Penedo et al. 1997; Penedo et al. 2009) \\
\hline LCamas (Las Camas) & LCamas (EHI) & (Urbina et al. 2007) \\
\hline LCan (La Cantueña) & LCan (EHI) & (Sanguino et al. 2007b) \\
\hline LCape (La Capellana) & LCape (EHI) & (Blasco y Baena 1989; Blasco et al. 1993) \\
\hline LCuad (La Cuadrá) & LCuadI (EHI) LCuadII (EHII) & (ARTRA 2010) \\
\hline LDese (La Deseada) & LDese (EHI) & (Martín y Virseda 2005; Martín 2007a) \\
\hline LEspe (Las Esperillas) & LEspeI (EHI) LEspeII (EHII) & (García y Encinas 1987, 1988) \\
\hline LGav (La Gavia) & $\begin{array}{l}\text { LGavI (EHII) LGavII (EHII) } \\
\text { LGavIII (EHII) } \\
\end{array}$ & (Quero et al. 2006) \\
\hline LLHo (Llano de la Horca) & LLHo (EHII) & $\begin{array}{l}\text { (Cerdeño et al. 1992; Baquedado et al. 2007; } \\
\text { Märtens et al. 2009) }\end{array}$ \\
\hline LMad (Las Madrigueras) & \begin{tabular}{|l|} 
LMadI (EHI) LMadII (EHI) \\
LMadIII (EHII) LMadIV (EHII) \\
\end{tabular} & (Almagro 1969) \\
\hline LPin (Los Pinos) & LPin (Transición EHI-EHII) & (Muñoz y Ortega 1996) \\
\hline LRibe (La Ribera) & LRibe (EHII) & (Galindo y Sánchez 2007) \\
\hline MValde (Mojón de Valdezarza) & MValde (EHII) & (Pérez y Bueno 2007b) \\
\hline PLJar (Puente Largo del Jarama) & PLJar (EHI) & (Muñoz y Ortega 1997) \\
\hline PMoros (Plaza de Moros) & PMoros (EHII) & (Urquijo y Urbina 2001; Urbina y Urquijo 2004) \\
\hline PP (Palomar de Pintado) & $\begin{array}{l}\text { PPI (EHI) PPII (EHII) PPIII (EHII) PPIV } \\
\text { (EHII) PPV (EHII) }\end{array}$ & $\begin{array}{l}\text { (Carrobles y Ruiz 1990; Pereira et al. 2001; } \\
\text { Pereira } \text { et al. 2003; Ruiz et al. 2004) }\end{array}$ \\
\hline Red5,25 (Redueña 5,25) & Red5,25 (EHII) & (Martín 1996; Alfaro y Martín 1996) \\
\hline SctIII (Sector III de Getafe) & SctIII (EHI) & (Blasco y Barrio 1986) \\
\hline SMaría (Santa María) & SMaría (EHI) SMaría (EHII) & (Pérez y Bueno 2002, 2007a) \\
\hline
\end{tabular}




\section{Notas}

1. Me gustaría agradecer a mis evaluadores sus aportaciones y críticas. Su trabajo ha mejorado sustancialmente el texto, aunque cualquier error, por supuesto, debe serme atribuido a mí.

2. La tabla de datos completa en la que se se describen los yacimientos asociados a sus respectivas variables puede consultarse en la versión digital de la revista Complutum, en el enlace http://revistas.ucm.es/index.php/CMPL.

\section{REFERENCIAS Bibliográficas}

Alfaro, M. y Martín, A. (1996): Restos celtibéricos en el término municipal de Redueña (Madrid). Boletín de la Sociedad Española de Amigos de la Arqueología, 36: 91-106.

Almagro, M., (1969): La necrópolis de "Las Madrigueras". Carrascosa del Campo (Cuenca). Bibliotheca Praehistorica Hispana, X, Madrid.

Almagro, M., (1976): Informe sobre las excavaciones en el Ecce Homo, Alcalá de Henares (Madrid). Noticiario Arqueológico Hispánico, 5: 293-300.

Almagro, M. y DÁvila, A.F. (1988): Estructura y reconstrucción de la Cabaña “Ecce Homo 86/6". Espacio, Tiempo y Forma. Serie I: Prehistoria, 1: 361-374.

Almagro, M. y Fernández-Galiano, D. (1980): Excavaciones en el Ecce Homo (Alcalá de Henares, Madrid). Diputación Provincial de Madrid, Madrid.

ARTRA, S.L. (2010): Memoria final de la Excavación arqueológica para el proyecto "Refuerzo del abastecimiento a Aranjuez y su zona de influencia desde la conducción Almoguera - Algodor”. Tramo 3-2. Yacimiento 4: La Cuadrá. Informe inédito.

Baquedado, E.; Contreras, M.; Märtens; G. y Ruiz, G. (2007): El oppidum carpetano de "El Llano de la Horca" (Santorcaz, Madrid). Zona Arqueológica, 10(2): 374-394.

Blánquez, J. (1999): Las necrópolis ibéricas en Castilla-La Mancha. Primeras Jornadas de Arqueología Ibérica en Castilla - La Mancha, Toledo: 49-87.

Blasco, M.C. (2007): El tránsito del Bronce Final al Hierro Antiguo en la cuenca baja del Manzanares. Zona Arqueológica, 10(1): 64-87.

Blasco, M.C. y Alonso, M.A. (1985): Cerro Redondo. Fuente el Saz del Jarama. Excavaciones Arqueológicas en España, 143, Madrid.

Blasco, M.C.; Alonso, M.A. y Lucas, M.R. (1991): Excavaciones en el poblado de la Primera Edad del Hierro del Cerro de San Antonio. Arqueología, Paleontología y Etnografía, 2: 7-187.

Blasco, M.C. y Baena, J. (1989): El yacimiento de La Capellana (Pinto, Madrid). Nuevos datos sobre las relaciones entre las costas meridionales y la Submeseta sur durante la Primera Edad del Hierro. CuPAUAM, 16: $211-231$.

Blasco, M.C.; Baena, J.; Millán, M.A.; Beneítez, P.; España, E. y Calderón, T. (1993): El Hierro Antiguo en el Alto Tajo. Aproximación cultural y marco cronológico apoyado en cuatro fechas de Termoluminiscencia del yacimiento de La Capellana. Madrider Mitteilungen, 34: 48-69.

Blasco, M.C. y Barrio, J. (1986): Excavaciones de dos nuevos asentamientos prehistóricos en Getafe (Madrid). Noticiario Arqueológico Hispánico, 27: 77-142.

Blasco, M.C.; Barrio J. y Pineda, M.P. (2007): La revitalización de los ritos de enterramiento y la implantación de las necrópolis de incineración en la cuenca del Manzanares: la necrópolis de Arroyo Butarque. Zona Arqueológica, 10(2): 215-238.

Blasco, M.C.; Carrión, E. y Planas, M. (1998): Datos para la definición de la Edad del Hierro en el ámbito carpetano: el yacimiento de Arroyo Culebro. CuPAUAM, 25.1: 245-281.

Bonet, H. y Mata, C. (2008): Las cerámicas ibéricas. Estado de la cuestión. Cerámicas hispanorromanas. Un estado de la cuestión (D. Bernal y A. Ribera eds.), Universidad de Cádiz, Cádiz: 147-170.

CArrasco, G. (ed.) (2007): Los pueblos prerromanos en Castilla-La Mancha. Universidad de Castilla - La Mancha, Cuenca.

Carrobles, J. y Ruiz, G. (1990): La necrópolis de la Edad del Hierro de Palomar de Pintado (Villafranca de los caballeros, Toledo). Actas del primer congreso de arqueología de la provincia de Toledo, Talavera de la Reina: $237-258$.

Cerdeño, M.L.; Martín, E.; Marcos, F. y Ortega, J. (1992): El yacimiento prerromano de Santorcaz (Madrid). Arqueología, Paleontología y Etnografía, 3: 131-167.

Consuegra, S. y DíAz-Del-Río, P. (2007): El yacimiento de La Albareja (Fuenlabrada, Madrid): un ejemplo de poblamiento disperso en la Edad del Hierro. Zona Arqueológica, 10(2): 131-152. 
Cuadrado, E. (1991): El castro de la Dehesa de la Oliva. Arqueología, Paleontología y Etnografía, 2: 191-255.

FernÁndeZ, V.M. (1988): El asentamiento ibérico del Cerro de las Nieves (Pedro Muñoz, Ciudad Real). I Congreso de Historia de Castilla - La Mancha. Talavera de la Reina: 359-369.

FERnÁNDEZ, V.M. y GARCíA, M. (1991): El tratamiento informático de datos funerarios cualitativos: análisis de correspondencias y algoritmo ID3 de Quinlan. Aplicaciones Informáticas en Arqueología (V.M. Fernández y G. Fernández, eds.), Complutum 1, Madrid: 121-131.

Fernández, V. M. y Hornero, E. (1988): Análisis funcional de los recintos domésticos del poblado ibérico del Cerro de las Nieves, Pedro Muñoz, Ciudad Real. Espacio y organización social. Estudios de Geografia e Historia: $163-178$.

Fernández, V.M.; Hornero, E. y Pérez, J.A. (1994): El poblado ibérico del “Cerro de las Nieves” (Pedro Muñoz). Excavaciones 1984-1991. Jornadas arqueológicas de Ciudad Real en la UAM. Universidad Autónoma de Madrid, Madrid: 111-129.

Galindo, L. y SÁnchez, V.M. (2007): El yacimiento carpetano de “La Ribera”. Zona Arqueológica, 10(2): 269289.

García, A.A. y Encinas, M. (1987): La necrópolis de la Edad del Hierro de "Las Esperillas", Santa Cruz de la Zarza (Toledo). Carpetania, I: 43-68.

García, A.A. y Encinas, M. (1988): Cerámicas incisas del conjunto funerario 44 - 45 de la necrópolis "Las Esperillas" (Santa Cruz de la Zarza, Toledo). Actas del Congreso sobre necrópolis celtibéricas, Daroca: 317-326.

GonzÁlez, C. (1999): Fíbulas en la Carpetania, DECEX S.L., Madrid.

Guinea, M. y Heras, C. (1991): Algunos ejemplos de la aplicación del análisis de correspondencias en arqueología. Aplicaciones Informáticas en Arqueología (V.M. Fernández y G. Fernández, eds.), Complutum 1, Madrid: 113-122.

Gutiérrez, E.; Muñoz, E.; Morlote J.M. y Montes, R. (2007): El horno de La Alberquilla: un centro productor de cerámica carpetana en Toledo. Zona Arqueológica, 10(2): 303-323.

Jensen, C.K. y Nielsen, K.H. (1997a): Burial data and correspondence analysis. Burial and society. The chronological and social analysis of archaeological data. En Jensen y Nielsen 1997: 29-62.

JENSEN, C.K. y Nielsen, K.H. (1997b): Burial and society. The chronological and social analysis of archaeological data. Aarhus University Press, Aarhus.

Kendall, D. G. (1971): Seriation from abundance matrices. Mathematics in the Archaeological and Historical Sciences (F. R. Hodson, D. G. Kendall, and P. Tautu, eds.), Edinburgh: Edinburgh University Press: $215-252$.

López, L.; Madrigal, A.; Muñoz, K. y Ortiz, J.R. (1999): La transición Bronce Final-Edad del Hierro en la cuenca media del Tajo: El yacimiento de Camino de las Cárcavas (Aranjuez, Madrid). II Congreso de Arqueología Peninsular, Zamora: 141-152.

Llopis, S. (1950a): La cerámica procedente de la necrópolis celtibérica de Villanueva de Bogas. Memoria de los Museos Arqueológicos Provinciales, IX-X (Extractos): 330-333.

Llopis, S. (1950b): Necrópolis celtibérica de Villanueva de Bogas (Toledo). Archivo Español de Arqueología, 78: $196-198$.

Märtens, G.; Contreras, M.; Ruiz, G. y Baquedado, E. (2009): El Llano de la Horca (Santorcaz). Un asentamiento carpetano en los albores de la romanización. Actas de las Terceras Jornadas de Patrimonio Arqueológico de la Comunidad de Madrid. Madrid: 210-222.

Martín, A. (1996): Excavación arqueológica de urgencia en torno al Km. 5,250 de la CN-320. Término municipal de Redueña (Madrid). Reunión de Arqueología Madrileña, Madrid: 216-218.

Martín, A. (2007a): Espacios domésticos y de almacenaje en la confluencia de los ríos Jarama y Manzanares. Zona Arqueológica, 10(2): 26-41.

Martín, A. (2007b): La necrópolis de El Vado (La Puebla de Almoradiel, Toledo): nuevos datos sobre el mundo funerario en época carpetana. Zona Arqueológica, 10(2): 255-268.

Martín, A. (2010): El hábitat carpetano y la necrópolis de El Vado (La Puebla de Almoradiel, Toledo). Actas de las II Jornadas de Arqueología de Castilla-La Mancha. Toledo, 309-342.

Martín, A. y VirsedA, L. (2005): Espacios domésticos y de almacenaje en la confluencia de los ríos Jarama y Manzanares. Encuentro de Jóvenes Investigadores sobre Bronce Final y Edad del Hierro en la Península Ibérica. Salamanca: 181-206.

Martín, A. y Walid, S. (2007): El yacimiento de El Baldío (Torrejón de Velasco, Madrid): algunos aspectos acerca de la evolución de los espacios de habitación entre los siglos V y I a. C.: de la cabaña al edificio. Zona Arqueológica, 10(2): 194-214. 
Miguel, J. DE y Morales, A. (1994): Informe sobre los restos faunísticos recuperados en el yacimiento de "El Cerrón”, Illescas, (Toledo). Excavaciones arqueológicas en “El Cerrón”, Illescas (Toledo): 206-211.

Montero, I. y Sejas, G. (2003-2004): Metales de la Dehesa de la Oliva (Patones. Madrid). Boletín de la Asociación Española de Amigos de la Arqueología, 43: 171-179.

Muñoz, K. y Ortega, J. (1996): La transición Primera - Segunda Edad del Hierro en el Bajo Henares. Las cabañas de "Los Pinos" (Alcalá de Henares, Madrid). Actas del V Encuentro de historiadores del Valle del Henares. Guadalajara: 31-41.

Muñoz, K. y Ortega, J. (1997): Elementos de inspiración orientalizante en la cuenca media del río Tajo: el yacimiento de "Puente Largo de Jarama" (Aranjuez, Madrid). SPAL, 6: 141-163.

Oñate, P.; Sanguino, J.; Penedo, E. y de Torres, J. (2007): El Caracol: un yacimiento de transición en la Primera Edad del Hierro madrileña. Zona Arqueológica, 10(2): 176-193.

Ortiz, J.R.; Madrigal, A.; López, L. y Muñoz, K. (2007): Camino de las Cárcavas (Aranjuez): desde el Hierro antiguo hasta los carpetanos. Zona Arqueológica, 10(2): 42-70.

Penedo, E.; Oñate, P. y Sanguino, J. (1997): El yacimiento de la “Laguna del Campillo". Un hábitat de la II Edad del Hierro en Rivas Vaciamadrid (Madrid). XXIV Congreso Nacional de Arqueología. Cartagena: 263-273.

Penedo, E.; Caballero C. y Sánchez-Hidalgo, F. (2002a): La ocupación de la II Edad del Hierro en el Arroyo Culebro (Leganés, Madrid). En VV.AA 2002: 71-124.

Penedo, E.; Orri, E.; Nadal, J.; Alonso, N.; Rovira, N.; Allué, E.; Font, J.; Reyes, M.; Enrich, J.; Arias, J.S. y Kotalla, L.R. (2002b): Análisis fisico-químicos. En VV.AA. 2002: 295-311.

Penedo, E.; Sánchez, M.; Martín, D. y Gómez, E. (2002c): La necrópolis de incineración de la Primera Edad del Hierro, En VV.AA 2002: 45-70.

Penedo, E.; Oñate, P.; Sanguino, J.; Pérez, D. y Bueno, M. (2009): El yacimiento carpetano de Laguna del Campillo (Rivas-Vaciamadrid). Actas de las Terceras Jornadas de Patrimonio Arqueológico de la Comunidad de Madrid. Madrid: 253-264.

Pereira, J.; Carrobles, J. y Ruiz, A. (2001): Datos para el estudio del mundo funerario durante la II Edad del Hierro en la Mancha Occidental: la necrópolis de Palomar de Pintado. Villafranca de los Caballeros (Toledo). II Congreso de Arqueología de la Provincia de Toledo. La Mancha Occidental y la Mesa de Ocaña (vol. 1). Toledo: 245-274.

Pereira, J.; Ruiz, A. y Carrobles, J. (2003): Aportaciones del C-14 al mundo funerario carpetano: la necrópolis de Palomar de Pintado. Trabajos de Prehistoria, 60(2): 153-168.

Pereira, J. (coord.) (2007): Prehistoria y protohistoria de la Meseta Sur (Castilla-La Mancha). Almud, Toledo.

Pérez, D. y Bueno, M. (2002): El yacimiento de Santa María. Historia 16, 311: 67-77.

Pérez, D. y Bueno, M. (2007a): El yacimiento arqueológico de Santa María, Villarejo de Salvanés (Madrid). Zona Arqueológica, 10(2): 324-341.

Pérez, D. y Bueno, M. (2007b): El yacimiento arqueológico del Mojón de Valdezarza (Villarejo de Salvanés, Madrid). Zona Arqueológica, 10(2), 395-411.

Quero, S.; Pérez, A.; Morín, J. y Urbina, D. (2005): El cerro de la Gavia. El Madrid que encontraron los romanos. Ayuntamiento de Madrid, Museo de San Isidro, Madrid.

Ringrose, T.J. (1992): Bootstrapping and correspondence analysis in archaeology. Journal of Archaeological Science, 19(6): 615-629.

Rodríguez, M., (2007): La fase carpetana de "El Malecón” (Madrid). Zona Arqueológica, 10(2): 290-302.

Rojas, J.M.; Garrido, G.; Gómez, A.J.; Guío, A. y Perera, J. (2007): El yacimiento de la I Edad del Hierro de Dehesa de Ahín (Toledo). Zona Arqueológica, 10(2): 71-106.

RuAno, E. (1996): Las cuentas de vidrio prerromanas del museo arqueológico de Ibiza y Formentera. Museu Arqueològic d'Eivissa i Formentera, Formentera.

Rubio, I. y Blasco, M.C. (2000): La Cronología del Hierro Antiguo en el área de Madrid a partir de los datos obtenidos por análisis de Termoluminiscencia. III Congresso de Arqueología Peninsular. Oporto: 225-236.

Ruiz, A.; Carrobles, J. y Pereira, J. (2004): La necrópolis de Palomar de Pintado (Villafranca de los Caballeros, Toledo). Investigaciones arqueológicas en Castilla - La Mancha, 1996 - 2002. Toledo: 117-133.

Ruiz, A. y Molinos, M. (1993): Los íberos. Análisis arqueológico de un proceso histórico. Crítica Arqueología, Barcelona. 
RuIz, G. (2007): Antes del Hierro. Cultura y sociedad en el centro de la meseta (ca. 1200 - 500 a.C.). Zona Arqueológica, 10(1): 37-62.

Ruiz, G.; Märtens, G.; Contreras, M. y Baquedano, E. (2012): Los últimos carpetanos. El oppidum de El Llano de la Horca (Santorcaz, Madrid). Museo Arqueológico Regional, Madrid.

Sanguino, J.; Oñate, P.; Penedo, E. y de Torres, J. (2007a): “El Colegio" (Valdemoro): cambios materiales y estabilidad socioeconómica a mediados del Primer milenio a.C. Zona Arqueológica, 10(2): 154-174.

Sanguino, J.; Oñate, P.; Penedo, E. y Torres, J. de (2007b): El yacimiento de la Primera Edad del hierro de "La Cantueña". Zona Arqueológica, 10(2): 108-118.

SAnguino, J.; Oñate P. y DE Torres, J. (2009): "El Colegio" (Valdemoro): evolución de un hábitat protohistórico a mediados del primer milenio a.C. Actas de las Terceras Jornadas de Patrimonio Arqueológico de la Comunidad de Madrid. Madrid: 223-231.

Santos, J.A., Perea, A. y Prados, L. (1998): El hábitat carpetano del Cerro del Gollino (Corral de Almaguer, Toledo). Iberia. Revista de la Antigüedad, 1: 53-72.

Torres, J. De (2012): La tierra sin límites. Territorio, sociedad e identidades en el valle medio del Tajo (S. IX - I a.C.). Tesis Doctoral. Universidad Complutense de Madrid, Madrid.

Torres, J. DE y Penedo, E. (2009): El lugar de cada uno: la necrópolis de Arroyo Culebro (Leganés) y la organización social de la EHI en la Carpetania. Actas de las I Jornadas de Jóvenes en Investigación Arqueológica. Dialogando con la cultura material. Madrid: 365-372.

Urbina, D. (1997): Espacio y cultura material del Hierro II en la Mesa de Ocaña. Tesis Doctoral inédita. Universidad Complutense de Madrid, Madrid.

Urbina, D. (1998): La Segunda Edad del Hierro en la Mesa de Ocaña. Un estudio regional de Arqueología del Paisaje. Arqueología Espacial, 19 - 20: 136-151.

Urbina, D. (2000): La Segunda Edad del Hierro en el centro de la Península Ibérica. Un estudio de arqueología espacial en la mesa de Ocaña (Toledo, España). BAR International Series, Oxford.

UrbinA, D. (2005): Recintos fortificados de la Segunda Edad del Hierro en el occidente de la provincia de Toledo. Espacios fortificados en la provincia de Toledo, Toledo: 41-68.

Urbina, D.; Morín, J.; Ruiz, L.A.; Agustí, E. y Montero, I. (2007): El yacimiento de Las Camas, Villaverde, Madrid Longhouses y elementos orientalizantes al inicio de la Edad del Hierro, en el valle medio del Tajo. Gerión, 25(1): 45-82.

Urbina, D. y UrquiJo, C. (2004): El poblado de la Edad del Hierro de Plaza de Moros, Villatobas (Toledo). Investigaciones arqueológicas en Castilla la Mancha, 1996-2002. Toledo: 75-88.

Urbina, D. y Urquijo, C. (2007): Cerro Colorado, Villatobas, Toledo: una necrópolis de incineración en el Centro de la Península. Zona Arqueológica, 10(2): 239-254.

Urbina, D.; Urquijo, C. y García, O. (2001): Hoyo de la Serna (Villarrubia de Santiago). El inicio de la Segunda Edad del Hierro en la Mesa de Ocaña. II Congreso de Arqueología de la Provincia de Toledo. La Mancha Occidental y la Mesa de Ocaña. Toledo: 85-109.

Urquijo, C. y Urbina, D. (2001): Plaza de Moros. Un recinto amurallado de la Segunda Edad del Hierro en la Mesa de Ocaña. II Congreso de Arqueología de la Provincia de Toledo. La Mancha Occidental y la Mesa de Ocaña. Toledo: 63-83.

VAliente, S. (1993): Cronología sobre el uso del torno y de la metalurgia del hierro en la Submeseta Sur (valle del Tajo), durante la II Edad del Hierro. Pátina. Homenaje a D. Raúl Amitrano, 6: 29-41.

VAliente, S. (1994): Excavaciones arqueológicas en "El Cerrón” de Illescas (Toledo), Servicio de Publicaciones de la Junta de Comunidades de Castilla-La Mancha, Toledo.

Vega, J.J.; Martín M.P. y Pérez, D. (2009): El poblado de la Segunda Edad del Hierro del Cerro de la Fuente de la Mora (Leganés, Madrid). Actas de las Terceras Jornadas de Patrimonio Arqueológico de la Comunidad de Madrid. Madrid: 281-290.

VV.AA. (2002): Vida y Muerte en Arroyo Culebro, Leganés. Museo Arqueológico Regional, Madrid.

VV.AA. (2007): Estudios sobre la Edad del Hierro en la Carpetania. Zona Arqueológica, 10. Museo Arqueológico Regional, Madrid.

VV.AA. (2008): El Primer Milenio a.C. en la Meseta Central. De la "longhouse al oppidum". Segundo Simposio AUDEMA, Madrid.

VV.AA. (2010): Actas de las Segundas Jornadas de Arqueología de Castilla - La Mancha. Diputación de Toledo, Toledo. 